\title{
Le tesi di laurea nelle biblioteche di architettura
}

a cura di

Serena Sangiorgi

Firenze University Press 
CNBA

Coordinamento Nazionale Biblioteche di Architettura

\section{Le tesi di laurea nelle biblioteche di architettura}

Giornata di studio Firenze, 28 maggio 2002

A cura di

Serena Sangiorgi

Firenze University Press

2003 
Le tesi di laurea nelle biblioteche di architettura : giornata di studio Firenze, 28 maggio 2002 / a cura di Serena Sangiorgi. - Firenze : Firenze University Press, 2003.

http:/ / digital.casalini.it/fulltext/is.asp?isbn=8884531020

Stampa a richiesta disponibile su http://epress.unifi.it

ISBN 88-8453-102-0 (online)

ISBN 88-8453-103-9 (print)

026.72 (ed. 20)

Biblioteche di architettura - Congressi

Giornata di studio organizzata da:

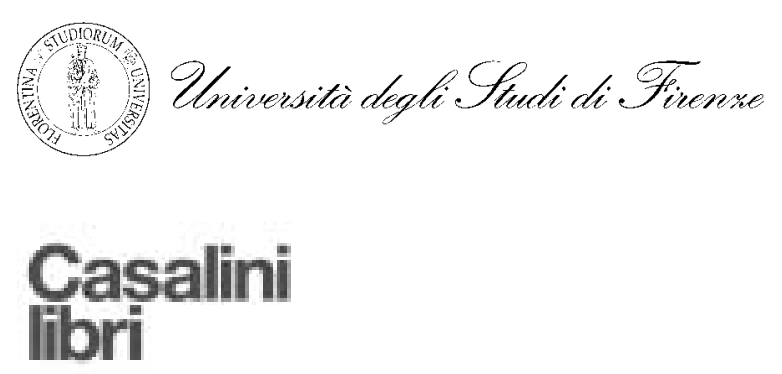

(C) 2003 Firenze University Press

Università degli Studi

Firenze University Press

Borgo Albizi, 28

50122 Firenze, Italy

http:/ /epress.unifi.it

e-mail:e-press@unifi.it 


\section{Indice}

Introduzione

Serena Sangiorgi

pp 1-4

Le tesi nel diritto d'autore: un argomento complesso

Antonella De Robbio pp. 5-13

Dalla biblioteca al centro documentale di ateneo:

un progetto per l'archivio delle tesi di laurea dell'Università di Firenze

Gianna Frosali pp. $15-24$

Dal supporto cartaceo al supporto digitale:

l'archivio delle tesi di laurea delle Facoltà di Architettura del Politecnico di Milano

Rossana Zucchinali pp. 25-34

Comunicare l'esperienza:

le tesi di laurea nella Biblioteca Centrale di Architettura

Alberta Zanella pp. 35-41

Il progetto Tesi-Meritorie della Facoltà di Architettura del Politecnico di Torino

Evandro Costa pp. $43-45$

Il progetto WebThesis

Daniela Ferrero pp. $47-50$

Progetto WebThesis: aspetti tecnici

Paolo Tealdi, Emanuele Venezia pp. 51-53

La gestione delle tesi in formato elettronico dello IUAV

Pierre Piccotti pp. 55-62

Tavola rotonda .pp. 63-64

Bibliografia .pp. $65-67$ 



\title{
Introduzione
}

\author{
Serena Sangiorgi \\ Biblioteca Politecnica \\ Università degli Studi di Parma
}

In occasione dell'assemblea annuale dei soci CNBA è divenuta consuetudine tenere un incontro su un tema i particolare interesse per le biblioteche di architettura: l'argomento scelto per il 2002 è stato la gestione delle tesi di laurea. La quasi totalità delle biblioteche associate ${ }^{1}$ è difatti legata alla realtà accademica e la mancanza, ancora a tutt'oggi, di una legislazione nazionale univoca e chiara per questo tipo di materiali ha portato ad una serie di varie esperienze locali, maturate in diversi contesti, che risultano di sicuro interesse per tutti, e in primo luogo per quelle realtà che non hanno ancora affrontato il trattamento delle tesi.

Proprio il fatto di non essere un problema risolto rende la gestione delle tesi una questione di attualità: per molto tempo la letteratura di riferimento è stata davvero scarsa ${ }^{2}$ e solo negli ultimi anni si sono viste pubblicazioni da cui trarre indicazioni operative ${ }^{3}$. La questione però è sentita da parecchi anni: negli archivi della lista AIB-CUR ${ }^{4}$, a cui molti soci sono iscritti, l'argomento ricorre a cadenza almeno annuale, e proprio all'inizio del mese di Maggio 2002 c'è stata una ripresa del tema, quasi a preparare questo nostro incontro fiorentino.

I problemi sono di molteplice natura, giuridici, tecnici, ma anche squisitamente biblioteconomici, cioè catalografici, conservativi e di consultazione: ogni struttura sembra aver messo a punto proprie procedure, a volte in un contesto ampio (di facoltà o di Ateneo), ma in assenza di un quadro legislativo uniforme le varie iniziative si presentano con caratteri di unicità.

\footnotetext{
${ }^{1}$ L'elenco dei soci è disponibile alla pagina <http: //www.cnba.it/soci.php>.

${ }^{2}$ Si veda BIB-Bibliografia Italiana di Biblioteconomia: $<$ http: / / www.aib.it/aib/bib/bib.htm $>$ : con la ricerca libera "tesi di laurea" vengono evidenziati 15 contributi, a partire dal 1991.

${ }^{3}$ Recentissimo, uscito poco dopo la giornata di Firenze del CNBA, è Pistelli, Zanetta - Zanon, Antonio, La catalogazione delle tesi, Roma, Associazione italiana biblioteche, 2002.

${ }^{4}$ Gli archivi sono disponibili ai soli iscritti alla lista: <http: //list.cineca.it/archives/aibcur.html>.
} 
La prima difficoltà evidenziata riguarda la consegna stessa della tesi, che non avviene con le stesse modalità né con gli stessi tempi: può essere prima o dopo la discussione, contemporaneamente o no alla consegna in segreteria, o sostituirsi addirittura ad essa, con conseguente necessità di preservazione dell'esemplare per il valore legale del titolo accademico.

Anche la forma dell'elaborato si presenta in varie modalità: può essere un esemplare in carta o su supporto magnetico, con o senza allegati (progetti, modelli in scala, videocassette, fotografie, tavole). I problemi tecnici sono quindi svariati, a partire dalla capacità/capienza del server stesso, che dovrà essere calcolata sulla base di un numero crescente di documenti digitali da gestire. Altre scelte importanti riguardano gli standard, diversi per documenti digitali alla nascita o digitalizzati a partire da supporti analogici tradizionali. In quest'ultimo caso sarebbe indispensabile prevedere una forza lavoro specializzata, ma almeno in una delle realizzazioni presentate si è dovuto ricorrere al supporto di studenti 150 ore, addestrati volta per volta, e non a personale specificamente addetto. Altra soluzione potrebbe essere l'affidamento delle procedure di digitalizzazione ad aziende esterne (outsourcing, pratica di utilizzo crescente per vari servizi all'interno delle biblioteche) oppure la creazione di consorzi tra realtà diverse.

Per alcuni progetti si hanno server dedicati alla versione digitale, che comportano gestioni complesse, ma non tutti con personale tecnico appositamente messo a disposizione.

Se in alcuni casi le tesi sono presenti almeno nel catalogo locale (in uno solo in quello di polo SBN, e limitatamente ad una sezione), più spesso figurano in cataloghi appositi e separati, con la conseguente necessità di spostarsi dall'OPAC dell'Ateneo per interrogare fonti diverse, con un notevole allungamento dei tempi di ricerca e un certo disorientamento dell'utente ben noto ai bibliotecari.

Non sempre il personale di biblioteca è chiamato ad aggiungere valore specifico a questi materiali con la catalogazione puntuale degli elaborati nelle loro diverse parti componenti, oppure con l'uso di metadati per i documenti digitali, che ne favoriscano l'identificazione e il reperimento (in una delle realtà sono i tesisti stessi a inserire i propri dati e quelli dell'elaborato, consentendo d'altro canto un notevole risparmio di tempo ai bibliotecari).

Per questi documenti infatti si pone, in fondo alla catena del trattamento, il problema della fruizione: probabilmente a causa della loro incerta natura non è scontata la disponibilità per prestito, neppure localmente, $\mathrm{o}$ per l'eventuale duplicazione (digitale o in fotocopia), o il prestito interbibliotecario. 
Dalla panoramica di esperienze emersa durante la giornata si evince che progetti nazionali come Thesis 995 non hanno visto l'adesione di tutti gli Atenei e Politecnici italiani, e soprattutto che il confronto con progetti internazionali (ad esempio Networked Digital Library of Theses and Dissertations $^{6}$, che prende le mosse da un progetto UNESCO del 1999) appare inesistente.

È dunque auspicabile in questo contesto attivare un'azione di informazione almeno tra i soci CNBA per portare a conoscenza dei vari modelli di intervento sinora attuati, nell'intento di dare suggerimenti utili alla gestione di un patrimonio documentale ancora oggi di difficile accesso.

Serena Sangiorgi

${ }^{5}$ Si veda: Archivio generale di ateneo, Università degli studi di Padova (a cura di), Thesis 99: progetto per la gestione, tenuta e tutela delle tesi di laurea, <http://www.unipd.it/ammi/archivio/ th_idx.htm>, ultimo agg. 2 giu. 1999. Padova, Università degli studi di Padova, 1996-1999

${ }^{6}$ Maggiori informazioni alla pagina <http: //www.ndltd.org > ; le ricerche sono possibili a partire dalla maschera di ricerca $<$ http $: / /$ hercules.vtls.com/cgi-bin/ndltd/chameleon $>$. 



\title{
Le tesi nel diritto d'autore: un argomento complesso
}

\author{
Antonella De Robbio \\ Biblioteca del Seminario Matematico \\ Centro di Ateneo per le Biblioteche \\ Università degli Studi di Padova
}

\begin{abstract}
"Gli uomini finiscono per essere così diversi solo perché hanno iniziato col copiare e perché continuano a farlo”.

Etienne Bonnot de Condillac

Essai sur l'origine des connoissances humaines, 1746
\end{abstract}

\begin{abstract}
La tesi, di laurea, di specializzazione, di master, di diploma, è opera dell'ingegno ed in quanto tale viene tutelata dalla legge. L'Autore della tesi rimane il solo e unico detentore dei diritti relativi alla medesima e non vi è alcun vincolo giuridico tra la tesi e il relatore. Primo tra tutti i diritto di pubblicazione, anello di congiunzione tra la sfera dei diritti morali e la sfera dei diritti economici. Un'eventuale pubblicazione in versione digitale delle tesi di laurea da parte di qualsiasi soggetto deve prevedere un'autorizzazione da parte dell'autore dell'elaborato stesso in quanto, se la digitalizzazione può essere lecita come azione ai fini di una conservazione o come prassi per documenti di tipo amminsitrativo, una pubblicazione richiede comunque un'autorizzazione. La tesi riveste una duplice personalità quale atto amministrativo e quale creazione originale di un autore, per questa sua caratteristica la materia va interpretata sotto diversi punti di vista.
\end{abstract}

La questione delle tesi nel diritto d'autore è materia assai complessa ${ }^{1}$, data la duplice natura dell'oggetto tesi nella sua qualità di:

- documento amministrativo o atto d'archivio

- documento scientifico protetto da diritto d'autore

\footnotetext{
${ }^{1}$ Per una trattazione estesa si rimanda agli atti della 2. Conferenza organizzativa degli archivi delle università italiane, tenutasi l' 11 e 12 novembre 1999 presso l' Università degli studi di Padova, pubblicati in Thesis 99: progetto per la gestione e tutela delle tesi di laurea, a cura di Gianni Penzo Doria, Padova, CLEUP, 2001.
} 
Altra domanda fondamentale: sono questi elaborati da considerarsi "pubblicazioni"?

Nella loro duplicità di ruoli le tesi devono essere indagate sotto due aspetti:

a) documento amministrativo che pone fine ad un procedimento mediante il quale lo studente consegue il diploma di laurea

b) opera originale dell'intelletto rientrante sotto tutela per il diritto d'autore

Un primo problema è quello relativo alla tesi come opera di ingegno. Sulla tutela giuridica delle tesi esistono solo tre pronunce giurisprudenziali (di cui due sullo stesso caso), come fa osservare il prof. Roberto Pennisi di Catania in uno dei suoi interventi al Gruppo padovano di studio Thesis99², gruppo costituitosi presso l'Università degli Studi di Padova ${ }^{3}$.

Di fatto è difficile stabilire sul piano probatorio chi abbia priorità sull'elaborato, ma questo se ci si ferma al contesto puramente gestionale: cioè la tesi nella sua qualità di prodotto/elaborato, intesa come atto amministrativo, atto dovuto verso l'Università, ai fini del conseguimento del titolo universitario. In questo caso ci si trova di fronte ad un documento amministrativo e necessariamente va trattato come un documento d'archivio.

Dall'altro versante, la seconda natura della tesi ci impone di indagare sulla tutelabilità o meno di questo prodotto a norma di legge. Va quindi esaminato cosa dice la legge a riguardo e va sottolineato che nessun regolamento di Ateneo può prevaricare i contenuti di legge.

All'interno degli atti normativi, i regolamenti hanno forza minore rispetto alle leggi.

Quello che può fare un Ateneo è, stabiliti i punti cardine dalla legge e precisamente se l'opera è originale o meno, e dopo aver individuato le fi-

\footnotetext{
${ }^{2}$ Per il progetto Thesis99 vedi contributo di Graziella Lunardi Il progetto Thesis99 in Thesis 99: progetto per la gestione e tutela delle tesi di laurea cit.

${ }^{3}$ Per una discussione ampia sulla questione delle tesi si veda il sito Thesis 99 dell'Archivio Generale di Ateneo - Università degli Studi di Padova che si prefigge di creare un sistema per la gestione, tenuta e tutela delle tesi di laurea <http://www.unipd.it/ammi/archivio/ th_idx.htm>
} 
gure aventi diritto, intervenire con 'autorizzazioni' a monte da far firmare allo studente all'atto dell'iscrizione all'Università.

Tali autorizzazioni servirebbero al fine di eventuali pubblicazioni (a stampa o in versione digitale) della tesi che verrà prodotta nell'ambito del 'rapporto' (di natura contrattuale) che si instaura tra lo studente e l'ente (l’Università) alla fine del percorso di studio.

Pennisi ci dice:

A livello di normativa secondaria o di regolamento di ateneo non si può modificare una normativa del regime dell'esclusiva. Infatti, è del tutto palese che la legge stabilisce tutta una serie di esclusive: brevetti, diritto d'autore ecc. Ciò esclude implicitamente che ce ne possano essere delle altre; l'esclusiva crea nel mondo economico e culturale una specie di barriera, oltre la quale non si può andare. Un'operazione che ne creasse altre ad hoc per le tesi di laurea o ampliasse esclusive già presenti o le modificasse in maniera sostanziale potrebbe essere compiuta solo mediante la legge.

E la legge ci dice implicitamente che le tesi in quanto opere intellettuali di carattere creativo rientrano sotto tutela di legge (disposizioni degli art. 1 e 2 della legge 633 del 1941 sul diritto d'autore).

La scarsa giurisprudenza che abbiamo a disposizione, in una delle tre sentenze citate da Pennisi ci dice che

la tesi non presenta carattere di originalità tale da integrare il requisito di proteggibilità. Questo carattere creativo viene negato non in relazione alla specifica tesi, ma a tutte indistintamente, perché esse sono frutto di collaborazione fra studente e relatore, il cui contributo è assai penetrante e talvolta decisivo.

Ma come osserva Pennisi e le altre due sentenze giurisprudenziali vi è un equivoco di fondo in questo ragionamento ed è quello che «la tesi ha carattere creativo o no sulla base di quello che è; si deve cioè esaminare il prodotto e poi verificare a chi spetti il diritto d'autore».

Come dice Marco Marandola, ricordandoci la legge 633/1941, tuttora in vigore, il carattere creativo di un'opera (e qui ci metto anche le tesi) nel diritto d'autore, non è protetto come idea, ma come forma espositiva dell'idea stessa.

$\mathrm{Si}$ accede alla tutela con uno sforzo intellettuale, anche se non grandissimo, ma che deve comunque essere in qualche modo originale: rielaborazione, riesposizione.

È il carattere di originalità che rende il lavoro tutelabile. 
Dato per quasi pacifico che la tesi rientra sotto tutela a chi spettano tutti i diritti, diritti che toccano la sfera morale, e i diritti esclusivi patrimoniali?

Nella tesi i contributi a vario titolo delle persone che vi interagiscono, non rientrano nella fattispecie di contributi indistinti e nemmeno si può parlare di opera di coautori, anche se questo aspetto, tutt'altro che secondario, può apparire a molti poco chiaro.

Soprattutto i relatori vorrebbero vedere il loro apporto riconosciuto al pari di quello dello studente, o quanto meno vantare dei diritti sull'elaborato.

È da considerare invece che le differenti tipologie di apporto intellettuale tra le due figure, quella dello studente e quella del relatore, hanno carattere fortemente diverso l'uno dall'altro: non si tratta di parti indivise della stessa opera, né tantomeno di capitoli separati redatti da ciascuno in separata sede.

È pur vero inoltre che l'apporto del relatore è molto variabile da Ateneo a Ateneo e da Facoltà a Facoltà e che quindi il coinvolgimento del relatore nella tesi dipende da più fattori e da varie condizioni.

Quale è il contributo 'originale' che va protetto nella tesi?

La legge sul diritto d'autore stabilisce che la protezione, come detto sopra non è riconducibile all'idea, ma alla forma che viene data a quell'idea. E la forma deve essere originale.

E veniamo al lavoro del relatore e cito sempre da fonte giurisprudenziale «semplici spunti, suggerimenti, o la comunicazione di un'idea non sono sufficienti a far ritenere che colui che ha dato gli spunti stessi sia coautore. Questa regola è un logico corollario di quella prima enunciata, secondo la quale il diritto d'autore non protegge un'idea, né tantomeno spunti e suggerimenti, ma protegge solo la forma espositiva» che deve essere operata dallo studente, altrimenti non può conseguire la laurea.

Lo studente deve essere in grado, alla fine del corso universitario, di operare un lavoro di confezionamento di un'idea trasmessagli dal relatore e di concretizzare tale idea in una "forma" creativa. 
Lo studente perciò raccoglie le indicazioni di relatori, tutor e altre figure che concorrono alla realizzazione del prodotto, coglie l'essenza intima dell'idea comunicata dal suo relatore e ne confeziona un prodotto originale di cui solo lui è l'autore e quindi titolare dei diritti morali e di tutti quelli esclusi.

Da notare che il ruolo del relatore è tra l'altro un ruolo istituzionale, che rientra nei compiti che il docente è tenuto a svolgere nell'ambito delle sue funzioni didattiche.

Il relatore non può essere considerato nemmeno coautore perché non deve (e non lo fa) occuparsi della forma dell'elaborato.

A questo si aggiunga che, visto e considerato che la tesi serve quale elaborato ai fini di una valutazione dello studente, come ci dice l'avv. Pennisi, il carattere peculiare dell'oggetto tesi è anche sancito in una legge a carattere penale e precisamente la legge 19 aprile 1925, n. 475 che reca un titolo assai eloquente: "Repressione della falsa attribuzione di lavori altrui da parte di aspiranti al conferimento di lauree, diplomi, uffici, titoli e dignità pubbliche”.

All'art. 2 tale legge prevede sanzioni per colui che procura o esegue per altri un lavoro che ha per scopo il conseguimento dei titoli di cui sopra, fra cui anche la laurea. Se così non fosse "tale operazione non sarebbe genuina e corretta" in quanto lo studente ha il dovere di elaborare personalmente la propria tesi.

Se il relatore ammette di aver conseguito un diritto d'autore sull'elaborato, lo può fare, ma in questo caso agisce "contra ius".

Altro aspetto non di minore importanza è in relazione al fatto se la tesi debba o no considerarsi pubblicazione.

Il tribunale di Perugia ha addotto, per escludere la tutelabilità della tesi di laurea nel diritto d'autore, il motivo per cui la tesi non è prodotto destinato alla pubblicazione o rivolto a un circuito commerciale. Ma si sa che anche le opere intellettuali inedite sono più che mai tutelate dal diritto d'autore, soprattutto in relazione al diritto all'inedito, diritto esclusivo rientrante nella sfera dei diritti morali dell'autore.

Il fatto che una tesi non venga considerata "pubblicazione" in quanto non pubblicata, non significa affatto che non rientri nella tutela.

La tesi, come abbiamo detto all'inizio, va vista nella sua duplice veste di: 
- documento amministrativo consegnato alla segreteria,

- "atto pubblico" orale, comunicato in sede di laurea (discussione della tesi),

ma può esiste anche in altra forma:

- quale documento non pubblicato, ma messo in circolazione all'interno dei servizi di biblioteca degli Atenei (consultazione e prestito o fotocopiatura).

In quest'ultimo caso essendo il documento non pubblicato rientra nella letteratura grigia, al pari di altri lavori come i rapporti tecnici o rapporti interni e i preprint scientifici.

Sia che si pensi alla tesi come documento amministrativo, o come atto pubblico orale o come letteratura grigia pervenuta in qualche modo in biblioteca, siamo di fronte a forme diverse dell'essere tesi e tutte e tre dal punto di vista giuridico, rientrano sotto tutela del diritto d'autore, in quanto si tratta di prodotto estrinsecato che viene posto di volta in volta in modi diversi.

Altro aspetto da considerare è che i testi delle pubblicazioni di fonte pubblica, gli atti ufficiali sono considerati di dominio pubblico e i documenti pubblici non rientrano sotto tutela, per il carattere di pubblicità.

Questa ulteriore complicazione è stata posta dal tribunale di Perugia, ma anche questo aspetto non può essere considerato valido nemmeno se vediamo la tesi solo come atto puramente amministrativo.

Come opera creativa intellettuale, avendo un autore non può essere considerata pubblicazione di fonte pubblica, per i motivi ampiamente spiegati sopra.

La tesi di Perugia secondo cui l'atto amministrativo perderebbe la tutelabilità, non regge in quanto vi è una pronuncia della Corte di appello stabilisce che la norma dell'art. 5 è eccezionale, «in quanto la tesi non è un atto ufficiale né dello stato né di un'amministrazione pubblica, quindi, anche ammesso che sia un documento amministrativo, non può essere ricompreso fra quei documenti».

Ricapitolando, ritorniamo a dire che : 
1) la tesi rientra nella tutela giuridica del diritto d'autore, su qualsiasi supporto essa sia confluita: la tutela si focalizza sulla forma data ad un'idea, filosofia cardine del diritto d'autore (tutela della forma e non del contenuto)

2) la tesi riveste una duplice personalità quale atto amministrativo e quale creazione originale di un autore

3) nella tesi l'avente diritto è lo studente. La sfera dei diritti morali è di pertinenza dello studente che redige la tesi il quale concreta l'idea del suo relatore in una forma tangibile

4) il relatore è portatore/comunicatore di un'idea ed è tenuto a seguire lo studente nella confezione di questa idea, in una forma tutelabile dalla legge quale creazione originale

Restano ancora in dubbio alcuni aspetti, non banali:

5) la tesi è o no una pubblicazione? Lo è certamente la sua versione pubblica comunicata oralmente in sede di discussione, ma chiaramente questa forma svanisce nel momento in cui viene discussa e quindi la sua tutelabilità si riconduce al diritto esclusivo di fissazione che l'oratore di un discorso detiene.

6) per una eventuale pubblicazione effettiva (a stampa o in formato digitale, la sostanza non muta) sarà necessario individuare chi è la figura che detiene i diritti economici. L'Ente in questo caso specifico potrebbe vantare dei diritti economici, visto e considerato che è stato possibile conseguire il titolo attraverso la produzione di un elaborato creato con le risorse e i mezzi dell'ente.

A maggior ragione nel caso in cui la tesi possa accedere ad un'esclusiva di tipo diverso, quella brevettuale per esempio, nel caso di lavori particolarmente originali con utilizzo di software nuovi (legge di tutela sul software), o laddove converga in una produzione di banche dati (legge di tutela sulle banche dati), non si può escludere che l'Ente possa vantare dei diritti economici, soprattutto in ragione dei due decreti legislativi sui programmi per elaboratore sulla tutela giuridica delle banche dati che attribuiscono all'Ente i diritti di sfruttamento economico per opere create dai lavoratori dipendenti. 
Nel caso della tesi, il rapporto tra lo studente e l'Ente non è lo stesso che intercorre tra il dipendente (anche a tempo parziale o a tempo determinato) e l'Ente, ma è pur sempre un rapporto tra le parti che produce dei risultati all'interno di un contesto definito e circoscritto.

Come argomenta Pennisi «tesi che raggiungano il risultato di dare una soluzione nuova ed originale ad un problema tecnico. Nel caso del brevetto è proprio l'idea che viene protetta, purché essa sia nuova. Un'idea è considerata nuova quando non fa parte dello stato della tecnica (inteso come l'insieme delle conoscenze che in un dato momento ci sono nella realtà tecnico-culturale) ed è esclusa dalla cosiddetta pre-divulgazione», nell'ottica di una tutela all'interno di un diritto assoluto diverso, quale per esempio una tutela brevettuale, essendo l'idea stessa in sé oggetto di tutela, e non la sua forma, lo studente perderebbe il suo ruolo di "autore".

Vi sono siti che danno la possibilità di pubblicare la propria tesi. Poichè tra i numerosi diritti che l'autore detiene vi è anche il diritto alla consultazione, diritto che prende il sopravvento qualora l'opera non sia considerata una vera a propria pubblicazione, questi siti offrono una consultazione delle tesi a pagamento, dietro autorizzazione dell'autore che ha richiesto la pubblicazione digitale dell'opera. Per acquisire il diritto alla consultazione integrale di una tesi, è sul sito "Tesionline.corriere.it" è necessario versare l'importo di Euro 18 IVA compresa .Tale importo è ripartito, a partire dalla seconda consultazione assoluta nell'anno in corso, al $50 \%$ tra l'Autore della tesi e Punto.it Srl, la società che gestisce il sito.

Sul sito viene riportata la seguente frase che riprende quanto sancito in merito dalla Corte d'Appello di Perugia:

La tesi di laurea costituisce un'opera dell'ingegno, come tale tutelabile in base alla normativa sul diritto d'autore, in quanto le attività di controllo, sorveglianza ed ingerenza, talvolta anche pregnanti, esercitate dal professore-relatore, non impediscono che la tesi sia il risultato precipuo dell'attività creativa del laureando. Sulla base di tali premesse, non si può certamente negare alla tesi di laurea il carattere di opera creativa, anche qualora il laureando utilizzi argomentazioni e temi già svolti da altri autori o si avvalga di consigli o delle ingerenze del professore-relatore, in quanto, anche in tal caso è individuabile almeno un "minimum" di elaborazio-

\footnotetext{
${ }^{4}<$ http://tesionline.corriere.it/faq/4.asp $>$
} 
ne ed organizzazione personale dello studente considerato sufficiente ai fini della tutelabilità del diritto d'autore.

Concludo sottolinenando che un'eventuale digitalizzazione delle tesi avrebbe un duplice versante di possibile "tutela" a due vie: da una parte diminuirebbe il plagio di tesi copiate da testi o da altre tesi, incrementando così la creazione di lavori davvero originali, dall'altra eviterebbe l'appropriazione indebita, da parte dei relatori, di pezzi consistenti di tesi, prodotte dai loro studenti. 



\title{
Dalla biblioteca al centro documentale di ateneo: un progetto per l'archivio delle tesi di laurea dell'Università di Firenze
}

\author{
Gianna Frosali \\ Biblioteca di scienze tecnologiche - Architettura \\ Università degli Studi di Firenze
}

\begin{abstract}
Presso la Biblioteca di Architettura dell'Università di Firenze è stato attivato un progetto relativo alle tesi di laurea che ha visto interventi sia per la catalogazione che per la conservazione e fruizione. La biblioteca si pone al centro del processo garantendo tutte le fasi della gestione.
\end{abstract}

\section{Dalla biblioteca...}

La Biblioteca di Architettura di Firenze cura la conservazione e la catalogazione delle tesi di laurea discusse in facoltà, in maniera sistematica dagli anni '70. In particolare in biblioteca è conservata la copia ufficiale dell'elaborato di tesi ai fini di ogni eventuale verifica o rilascio di certificazioni di conformità. Presso la biblioteca i laureati possono richiedere una copia autenticata della propria tesi di laurea per concorsi e borse di studio presso enti o fondazioni italiane e straniere che non possano accettare l'autocertificazione.

Prima degli anni ' 70 le tesi di laurea venivano consegnate alle segreterie studenti a cura del laureando prima della data prevista per la discussione, ma poiché questo comportava che il materiale di tesi consegnato fosse composto di pochi fogli sciolti e fossero spesso assenti gli elaborati grafici fece che, successivamente, la raccolta fosse fatta dalla presidenza di facoltà direttamente dalla sede di tesi. Quindi fin dalla nascita dell'archivio le tesi arrivavano in biblioteca per ogni sessione di laurea direttamente dalle sedi di tesi, per la catalogazione la conservazione e la consultazione.

La gestione dell'archivio delle tesi di laurea si può scomporre in quattro momenti importanti che ne determinano la struttura: consegna, catalogazione, conservazione, consultazione. 
La consegna della tesi di laurea è un aspetto gestionale che ha ricadute, per alcune facoltà abbastanza pesanti sulla conservazione e sulla consultazione dei documenti. La regolamentazione di questo momento è, quindi, il primo problema da affrontare.

Infatti, fin dall'inizio della costituzione dell'archivio tesi il materiale consegnato ha comportato problemi di collocazione sugli scaffali e quindi dagli anni '80 è iniziata la regolamentazione della consegna del materiale di tesi.

Inizialmente veniva richiesto di consegnare tutto il materiale allegato alla relazione piegato e raccolto in cartelle, successivamente siamo passati attraverso un periodo di transizione in cui i materiali allegati potevano essere sostituiti, a discrezione del laureando, da diapositive, o fotografie, o negativi fotografici, oggi obbligatoriamente i materiali cartacei allegati alla relazione di tesi devono essere sostituiti da diapositive, o fotografie, o negativi fotografici, o videocassetta (formato VHS), o CD-ROM.

Devono esserne consegnate due copie: una, 10 giorni prima dell'inizio di ogni sessione di laurea, per le esigenze della Commissione di tesi, presso la segreteria del Dipartimento, questa copia non è completa perché è prevista la consegna della sola relazione di tesi, l'altra, al momento della discussione della tesi, dove il laureando lascia l'elaborato di tesi completo degli allegati grafici in consegna al Presidente della Commissione tesi che provvederà poi alla consegna in biblioteca insieme con un elenco degli elaborati ricevuti, che attesta la corrispondenza tra i materiali discussi e quelli consegnati ed è questa la copia ufficiale.

Il modello di frontespizio, le indicazioni per la redazione delle tesi di laurea cui i laureandi devono attenersi per la preparazione degli elaborati e le modalità di consegna presso la biblioteca sono descritte sulla pagina web della biblioteca.

La relazione dattiloscritta deve essere nel formato A4, firmata sul frontespizio dal professore relatore di laurea e dal laureando o in formato elettronico (floppy, cartuccia zip, cd-rom) e gli elaborati grafici (tavole), solo in sede di tesi, dopo verifca della corretta conformità da parte del Presidente di Commissione, devono essere sostituiti da diapositive, o fotografie, o negativi fotografici o videocassetta (formato VHS), o CD-ROM.

La consegna della relazione di laurea avviene 10 giorni prima dell'inizio di ogni sessione di laurea, presso la segreteria del Dipartimento nel quale si discute la tesi. Tale consegna, vincolante ai fini della discussione della tesi, ha lo scopo di permettere ai docenti che fanno parte della Commissione degli esami di laurea di prendere preventivamente visione dei lavori pre- 
sentati: è quindi nell'interesse del laureando la consegna della relazione nella sua forma più completa possibile. Tale relazione verrà riconsegnata al laureando entro 30 giorni perché l'unica copia conservata della tesi di laurea è quella di cui al successivo punto 2. Il laureando è tenuto a contattare preventivamente il Dipartimento poiché alcuni non restituiscono la relazione.

La consegna definitiva degli elaborati di laurea (relazione e altro materiale allegato) avviene al momento stesso dell'approvazione dell'esame di laurea, nelle mani e sotto la responsabilità del Presidente di Commissione. Gli elaborati così consegnati, nella loro forma più completa, definitiva ed integrale, accompagnati da un elenco redatto dal Presidente di Commissione, sono gli unici ed i soli ad essere archiviati presso la biblioteca della facoltà: solo su di essi la biblioteca stessa potrà rilasciare eventuali certificazioni o autentiche che venissero richieste dagli interessati.

Gli elaborati che costituiscono il materiale per la consegna definitiva di cui al punto precedente dovranno essere redatti secondo le seguenti modalità:

- relazione nel formato corrente del foglio dattiloscritto (A4) recante sul frontespizio la firma del Professore relatore di laurea e del laureando. La relazione può essere consegnata anche in formato elettronico (floppy, cartuccia zip, cd-rom).

- elaborati grafici (tavole): unicamente in sede di tesi, dopo verifica della corretta conformità da parte del Presidente di Commissione, devono essere sostituiti da diapositive, o fotografie, o negativi fotografici, o videocassetta (formato VHS), o CD-ROM.

Tali modalità di consegna sono dettate dall'esigenza di poter archiviare tutto il materiale che fa parte integrante degli elaborati di laurea.

L'autorizzazione. Ci sono aspetti che, affrontati (o non affrontati), al momento della consegna della tesi incidono sulla consultazione delle tesi stesse ed uno di questi aspetti riguarda l'autorizzazione alla consultazione che fin dall'inizio la biblioteca di architettura ha richiesto all'autore.

$\mathrm{Al}$ momento della laurea il laureando può lasciare in biblioteca un'autorizzazione preventiva alla lettura della sua relazione di tesi; se l'autore non rilascia tale autorizzazione la tesi potrà essere consultata solo da coloro personalmente autorizzati. Dal 1995 la non autorizzazione vale solo per 5 anni, trascorso tale periodo, se il divieto di lettura non viene rinnovato, la tesi viene considerata autorizzata alla lettura. 
La catalogazione Il materiale dell'archivio tesi dall'anno accademico 1965-66 al 1984-85 è stato catalogato con le regole biblioteconomiche. Le schede cartacee delle tesi venivano raccolte in un catalogo separato, consultabile presso la Biblioteca, e ordinato per laureando, relatore e soggetto. Invece, dall'anno accademico 1985-86 fino al 1998 sono state catalogate in formato elettronico con una produzione cartacea di indici per anno accademico, per laureando, per relatore e soggetto.

Un progetto tesi del Sistema Bibliotecario di Ateneo ha portato nel 1999 all'immissione in rete dei titoli delle tesi discusse dall'anno accademico 1976-77. Il catalogo in linea contiene le informazioni relative alle tesi di laurea e di diploma discusse presso l'Ateneo dal 1976 (compresi dati parziali degli anni 1974 e 1975). I dati sono estratti dal sistema gestionale delle Segreterie Studenti.

Il catalogo delle tesi di laurea è quindi attualmente consultabile on-line e permette una ricerca per chiavi specifiche: titolo o parte di esso (parola chiave del titolo), autore, relatore, anno accademico, Facoltà.

La conservazione. La biblioteca è depositaria della copia dell'elaborato di tesi, conservata come documento ufficiale ai fini di ogni eventuale verifica o rilascio di certificazioni di conformità.

Come detto precedentemente il momento della consegna della tesi di laurea, è una fase importante per quello che riguarda successivamente la conservazione. Se si sono previste precedentemente delle modalità di consegna dettagliate sia per quanto riguarda la preparazione degli elaborati da consegnare sia per quanto riguarda il momento stesso della consegna la conservazione sarà facilitata . Relativamente al momento della consegna degli elaborati più la consegna degli elaborati di tesi si avvicina al momento della laurea, quanto più saremo in grado di conservare un documento conforme. Per quanto riguarda la facoltà di architettura dell'Ateneo di Firenze il laureando consegna l'elaborato di laurea al momento stesso della laurea nella forma più completa, definitiva ed integrale direttamente al Presidente della Commissione tesi che provvederà poi alla consegna in biblioteca. "Gli elaborati così consegnati, , accompagnati da un elenco redatto dal Presidente di Commissione, sono gli unici ed i soli ad essere archiviati presso la biblioteca della facoltà: solo su di essi la biblioteca stessa potrà rilasciare eventuali certificazioni o autentiche che venissero richieste dagli interessati”.

La consultazione. In assenza di un regolamento comune a livello d'Ateneo riguardante le norme per la lettura delle tesi, la Biblioteca si attiene 
a quanto previsto dalla legge sul diritto d'autore e dall'Art. 16 dell'attuale regolamento quadro per le biblioteche dell'Università di Firenze, che recita così:

Le tesi di laurea depositate presso la biblioteca possono essere date in visione solamente con l'autorizzazion dell'autore, o in caso di impossibilità del suo reperimento, del Preside, sulla base di motivata richiesta. La biblioteca, secondo le sue caratteristiche e necessità, adotta le forme più opportune per la visione delle tesi, tenuto conto del materiale della tesi e della salvaguardia del diritto d'autore (legge 1.633/41). È comunque esclusa la riproduzione di qualunque pagina parte della tesi data in visione.

Quindi per quanto riguarda la consultabilità delle tesi di architettura, come detto precedentemente, avendo fin dall'inizio la biblioteca richiesto all'autore l'autorizzazione alla consultazione, le tesi sono per la maggior parte consultabili.

Come detto precedentemente, dal 1995 avendo la "non autorizzazione" alla consultazione validità 5 anni, trascorso questo periodo se non viene rinnovata la tesi diventa consultabile.

Come regola generale si segnala comunque che la lettura è limitata alla relazione scritta, mentre è escluso dalla consultazione tutto il materiale allegato (tavole, diapositive, fotografie, etc.).

Le tesi di laurea possono essere solo consultate e non possono essere fotocopiate né riprodotte con qualsiasi altro mezzo. Fino al 1999, la Biblioteca consentiva la consultazione delle tesi solo agli studenti della facoltà. La richiesta di consultazione delle tesi, doveva essere firmata dal docente per cui veniva fatta la ricerca ed ogni richiesta era limitata a tre tesi. Attualmente le relazioni delle tesi di laurea autorizzate alla lettura possono essere consultate, presentando una lettera di richiesta-autocertificazione. La consultazione è riservata agli studenti iscritti, a tutto il personale docente e tecnico amministrativo e ai dottorandi, specializzandi e iscritti ai corsi di perfezionamento dell'Università degli Studi di Firenze, tutti gli altri utenti possono accedere alla lettura solo con l'autorizzazione scritta dell'autore con firma autenticata. Le tesi di laurea non autorizzate alla lettura possono essere consultate solo con l'autorizzazione scritta dell'autore, anche per consultare l'eventuale materiale allegato (tavole, diapositive, fotografie ecc.) o per fare delle copie è necessaria la preventiva autorizzazione scritta dell'autore con firma autenticata. 


\section{...al centro documentale d'Ateneo}

Presso il Sistema Bibliotecario dell'Università degli studi di Firenze relativamente agli archivi delle tesi di laurea è stato concluso un progetto per il triennio 1999-2001 che, nella prima fase, ha portato alla riunificazione dei molti fondi tesi ufficiali o meno presenti in Ateneo arrivando alla situazione attuale che conta fondi generalmente unici di facoltà.

Il riversamento dei i dati posseduti dalle segreterie studenti ha portato all'immissione in rete dei titoli delle tesi dell'intero Ateneo discusse dall'anno accademico 1976-77, creando un catalogo unico di ateneo.

La collocazione è automatica su base di ateneo o per facoltà il programma cioè prevede sia la collocazione unica di ateneo (una sorta di numero di ingresso) sia divisa per facoltà e per anno solare di discussione della tesi (non per anno accademico) Dal 1999, anche l'etichettatura è uniforme per tutto l'Ateneo, ed è in corso il recupero del pregresso.

Affrontato quindi,in questo primo progetto, l'aspetto catalografico il progetto successivo si propone di intervenire sui regolamenti nell'intento di normalizzare a livello di Ateneo le procedure di consegna, conservazione e consultazione delle tesi di laurea.

La duplicità della natura della tesi di laurea, che è da considerarsi sia atto amministrativo, in quanto documento finale e legale del corso di studio, sia materiale documentario culturale e scientifico farebbe sì che una copia fosse conservata nel fascicolo dello studente e un'altra per la consultazione fosse depositata nelle biblioteche. La situazione dell'Università degli Studi di Firenze è anomala in quanto l'unica copia esistente considerata ufficiale è quella che è conservata nelle biblioteche quindi il progetto tesi per il biennio 2002-2003 si propone la nascita di un Centro documentale di Ateneo per il trattamento, la conservazione, la tutela e la consultazione della documentazione archivistica e delle tesi di laurea, dove le tesi potranno essere gestite sia come documento amministrativo sia come documento scientifico. Essendo le tesi di laurea una parte importante del complesso dei documenti che un archivio universitario è tenuto a gestire e conservare il progetto propone la creazione di un sistema per la gestione, delle tesi di laurea dell'ateneo.

La realizzazione del progetto che prevede la creazione di un centro di documentazione in grado di valorizzare e, nello stesso tempo, liberare le biblioteche di area da una mole notevole di materiale che, fino ad oggi, si è rivelato poco gestibile con le loro sole energie, dovrebbe permettere l'unificazione di tutti i fondi tesi con la conseguente normalizzazione delle regole sia per quanto riguarda la consegna del materiale di tesi sia per la 
consultazione dei documenti. Il posseduto delle biblioteche di area, ammonta complessivamente, al dicembre 2001, a circa 3500 metri lineari, da incrementarsi, su base annua, di circa $250 \mathrm{ml}$ anche se la previsione sarà da rivedere e rispetto al nuovo ordinamento universitario.

Il passo successivo naturale che ci si pone oggi, è dunque la definizione di un percorso di gestione e consultazione del materiale e il suo accorpamento in tutto o in parte, in un'unica sede fisica definito da un progetto per l'allestimento di un centro per il trattamento, la conservazione, la tutela e la consultazione della documentazione archivistica e delle tesi di laurea.

È chiaro che l'intero progetto avrà comunque bisogno di essere recepito a livello di regolamenti d'Ateneo.

In base al reperimento degli spazi potranno essere attuate due soluzioni, un'ottimale in cui tutto il patrimonio relativo alle tesi di laurea di tutto l'Ateneo è conservato nella sede del Centro documentale di Ateneo, qualora invece gli spazi non fossero sufficienti per l'attuazione di questa soluzione, le tesi dell'Ateneo troverebbero localizzazione in due sedi: Biblioteca scienze sociali, per le tesi relative all'area delle scienze sociali e Centro documentale di Ateneo per le tesi relative all'area biomedica, umanistica, scientifica e tecnologica.

La consegna. Attualmente, per quanto concerne le tesi di laurea, le modalità di presentazione, conservazione e consultazione differiscono notevolmente all'interno dell'Ateneo da facoltà a facoltà, come d'altra parte in molti degli atenei del territorio nazionale.

Le tesi arrivano alle biblioteche, dalle segreterie studenti, dalle commissioni di laurea, dalle presidenze di facoltà, dai laureandi stessi, insomma nei modi più disparati, Perciò è necessario definire un percorso uniforme, che permetta di mantenere il controllo sul materiale con relativa semplicità. Serve quindi creare uno standard per la consegna degli elaborati di tesi.

Il progetto così prevede la normalizzazione della gestione amministrativa:

- definizione del percorso da seguire per depositare la copia ufficiale della tesi nell'archivio

- inserimento, nella domanda di tesi che il laureando compila all'atto della presentazione della tesi di laurea, dell'autorizzazione o meno alla consultazione della stessa. (Attualmente solo alcune facoltà richiedono quest'informazione e la utilizzano come liberatoria). 
- implementazione del campo autorizzazione nel data-base a disposizione delle segreterie studenti.

È necessaria una riflessione per quanto riguarda la definizione del percorso da seguire per depositare la copia ufficiale della tesi nell'archivio: molti atenei, che già da tempo hanno iniziato un percorso normalizzato e uniforme di trattamento delle tesi, identificano le segreterie studenti come punto di arrivo della copia ufficiale. Ma certo è, che la consegna molto anticipata della tesi di laurea, riducendo i tempi a disposizione del laureando può portare, per alcune facoltà, ad avere una difformità tra ciò che viene consegnato alle segreterie studenti e la versione finale dell'elaborato che verrà discusso in sede di tesi. Solo la consegna il giorno stesso della discussione, direttamente al Presidente della Commissione, potrebbe assicurare che l'elaborato che l'Ateneo conserverà come copia ufficiale sarà effettivamente l'ultima versione. Ma è vero anche che la commissione di tesi deve avere prima della discussione a disposizione una copia completa e non solo una bozza da visionare. Perciò sarà necessario definire un percorso uniforme che permetta di mantenere il controllo sul materiale con relativa semplicità. Se la scelta fosse indirizzata sulla soluzione che prevede la consegna il giorno stesso della discussione, le tesi di laurea dovranno essere raccolte nel corso delle varie sessioni di laurea dalle varie sedi e successivamente riunite nel centro documentale, che sarà incaricato della loro gestione, conservazione e valorizzazione. Comunque sarà necessario sciogliere i nodi relativi al tempo di consegna della tesi per poter risolvere anche molti dei successivi problemi relativi alla conservazione.

Per quanto riguarda l'autorizzazione o meno alla consultazione della tesi dobbiamo tener presente che, riconosciuta al laureando la paternità intellettuale dell'elaborato di tesi, questo va tutelato come opera dell'intelletto sia evitandone la riproduzione sia controllandone la consultazione è quindi indispensabile che il laureando stesso autorizzi o no la consultabilità della propria tesi di laurea per poter essere tutelato nel caso di una pubblicazione scientifica.

La conservazione. Le tesi di laurea sono materiale destinato alla conservazione perenne, quindi va tutelato l'originale depositato nell'archivio universitario. Il Centro documentale dovrebbe diventare depositario giuridico e gestore scientifico delle tesi di laurea sia per creare un fondo unico di Ateneo gestito con criteri generali e normalizzati sia per la conservazione della memoria culturale dell'Ateneo. La tesi di laurea va conservata in archivio assieme agli altri documenti che formano il fascicolo dello 
studente, così come viene già fatto in altri Atenei. $\grave{E}$ comunque preferibile conservare la tesi di laurea in archivio in un luogo fisicamente separato dal fascicolo dello studente, ordinata per facoltà e all'interno per anno di discussione insieme a tutto il corpus delle tesi. Con l'inventariazione e l'informatizzazione dell'archivio di deposito e in particolare dell'archivio studenti potrebbe essere ricostruita la carriera universitaria con un rimando dal catalogo tesi al fascicolo dello studente e viceversa.

Come ho più volte ripetuto le attente modalità di consegna porteranno ad avere nell'archivio universitario degli esemplari per la conservazione che corrispondono a quelli effettivamente discussi in sede di tesi di laurea, perché non si conservino esemplari non completi e si debba poi fare su questi certificazioni di conformità all'originale.

Considerando l'aspetto della conservazione delle tesi sarà necessario affrontare il problema della corretta conservazione dei materiali di tesi prodotti in formati non standard, come possono essere le cassette audiovideo o i cd-rom per poterli tramandare come futura memoria.

La consultazione. Attualmente all'interno dell'Ateneo fiorentino la consultazione delle tesi di laurea varia da biblioteca a biblioteca, ma chiarita la natura delle tesi e riconosciuta la paternità intellettuale del laureando, sarà necessario tutelare gli elaborati di tesi definendo criteri e limiti della consultabilità. Per questo il progetto tesi prevede l'elaborazione di un regolamento sulle modalità di tenuta e di consultazione delle tesi di laurea che stabilisca regole comuni di consultazione sulla base del diritto esistente in materia, analizzando anche le scelte operate in altre realtà italiane ed europee, che consenta la consultazione della tesi per motivi di studio senza inutili burocratismi ma che comunque la salvaguardi da un uso improprio.

Sarà quindi necessario che venga definito un regolamento di consultazione, da integrarsi al regolamento del Sistema Bibliotecario d'Ateneo e alla carta dei servizi.

La valorizzazione. Rimane ancora da affrontare, per il futuro, l'aspetto della valorizzazione delle tesi di laurea. Ogni università dovrebbe considerare questi documenti molto importanti, in quanto prodotto in grado di misurare il suo stato di eccellenza.

Le tesi per essere valorizzate e al tempo stesso salvaguardate, devono essere conosciute, infatti il miglior antiplagio è proprio la pubblicizzazione. In Italia questo patrimonio non è sufficientemente pubblicizzato e l'assenza d'informazione favorisce il plagio. 
A questo scopo forse potrebbe bastare avere in rete, insieme all'informazione catalografica, l'abstract e le notizie bibliografiche, anche nella prospettiva di creazione della biblioteca digitale.

Auspicabile sarebbe la creazione di un catalogo unico nazionale, come hanno alcuni stati anche europei (senza voler parlare sempre degli Stati Uniti d'America), che potesse permettere la massima diffusione anche come contributo alla ricerca scientifica. 


\title{
Dal supporto cartaceo al supporto digitale: l'archivio delle tesi di laurea delle Facoltà di Architettura del Politecnico di Milano
}

\author{
Rossana Zucchinali \\ Centro di Documentazione dell'Architettura e del Territorio \\ Politecnico di Milano
}

\begin{abstract}
Presso il CEDAR (Centro di Documentazione dell'Architettura e del Territorio) sono raccolte dal 1978 le tesi di laurea delle Facoltà di Architettura del Politecnico di Milano. L'archivio consta di oltre 19.000 tesi, con un incremento annuo di circa 1.400 documenti. Il presente contributo descrive le diverse fasi della gestione dell'archivio esplicandone la duplice funzione: archiviazione della tesi in quanto documento amministrativo conclusivo del corso di laurea e trattamento biblioteconomico finalizzato a rendere disponibile -nel rispetto e nella tutela del diritto d'autore- a studenti, docenti, ricercatori e studiosi, un materiale documentale spesso di difficile accesso presso gli atenei italiani. Sono quindi illustrate le modalità di consegna e raccolta delle tesi, la gestione amministrativa, la catalogazione, le regole per la consultazione. La seconda parte del'intervento verte sul progetto di digitalizzazione delle tesi di laurea, attivato nel 1998 su indicazione del Consiglio di Facoltà, volto alla creazione di un sistema di consultazione informatizzato - in rete locale - con l'obiettivo di potenziare il servizio in termini di rapidità di accesso e di protezione dei materiali conservati. Sono quindi analizzate le problematiche legate allo sviluppo del progetto: dallo studio di fattibilità alle fasi di sperimentazione, dalla scelta dei formati presi a standard al sistema di ricerca per la consultazione di un archivio costituito ad oggi da circa 1.500 tesi.
\end{abstract}

\section{Formazione dell'archivio e sue finalità}

«Dal momento della sua costituzione, nel 1977, il CEDAR svolge nell'ambito della documentazione e della ricerca documentale un'attività che privilegia la formazione, l'aggiornamento e la catalogazione automatizzata di collezioni e di archivi relativi a materiali non reperibili nei normali circuiti di pubblicazione» ${ }^{1}$.

Proprio in questa ottica, intorno alla fine degli anni ' 70 , poco doco la fondazione del CEDAR, si cominciò a pensare alla raccolta delle tesi di

${ }^{1}$ Dalla nota di presentazione del CEDAR <http://www.cedar.polimi.it $>$ 
laurea della Facoltà di Architettura per la creazione di un archivio che si ponesse come obiettivo la conservazione e la diffusione degli elaborati di tesi come materiale documentario aggiuntivo rispetto alle classiche fonti offerte a studenti e studiosi.

In precedenza le tesi venivano consegnate presso i dipartimenti o gli istituti creando quindi una situazione frammentata e quasi sicuramente una rilevante perdita di documentazione: a partire dal $1978 \mathrm{i}$ dipartimenti cominciarono a far confluire le tesi di cui erano in possesso nell'archivio che si andava costituendo.

Nel 1984, con la delibera del Senato Accademico che sanciva l'obbligo per i laureandi di consegnare gli elaborati presso il CEDAR, per l'archivio delle tesi si delineò in maniera istituzionalmente formalizzata la duplice funzione di custodia del documento amministrativo conclusivo del corso di laurea (anche ai fini del rilascio di certificazioni di conformità) e di diffusione di un materiale documentale spesso di grande interesse, ma altrettanto spesso di difficile accesso presso gli atenei italiani.

Se per i primi anni l'archivio può considerarsi certamente lacunoso, dalla metà degli anni ' 80 esso raccoglie tutte le tesi discusse presso le Facoltà di Architettura ${ }^{2}$ del Politecnico di Milano e ha una consistenza ad oggi pari a circa 19.000 documenti.

A quasi 25 anni dalla sua formazione, l'esperienza di gestione dell'archivio delle tesi di laurea costituito presso il CEDAR si rivela altamente positiva in quanto di riconosciuta utilità:

- per i docenti e i ricercatori del Politecnico: attraverso il catalogo in rete viene data pubblicità all'informazione bibliografica relativa all'attività didattica e di ricerca svolta dai docenti nelle vesti di relatore o correlatore la cui traccia si trova evidente nelle tematiche trattate dalle tesi;

- per i laureati: una tesi catalogata e messa a disposizione del pubblico è certamente valorizzata (fermo restando che spetta all'autore decidere se permettere o meno la consultazione del proprio elaborato);

- per i laureandi: la consultazione dell'archivio è prassi abituale per reperire indicazioni sui temi affrontati dai vari docenti, esempi dei diversi modi di impostare la tesi e di trattare gli argomenti, oltre naturalmente ad un'ampia fonte di spunti bibliografici;

- per gli studiosi di architettura e delle discipline affini: la collezione del-

${ }^{2}$ Le disposizioni sulla consegna delle tesi furono estese alla II Facoltà di Architettura Milano Bovisa e alla III Facoltà di Architettura Milano Bovisa (Disegno Industriale) istituite rispettivamente nel 1992 e nel 2000. 
le tesi ha raggiunto ormai una consistenza ragguardevole e costituisce una raccolta di materiale documentale di interesse scientifico variabile ma sicuramente unico ${ }^{3}$.

\section{Gestione dell'archivio}

\subsection{L'attività di gestione amministrativa}

Le norme generali per la consegna delle tesi prevedono che esse siano depositate presso il CEDAR entro una specifica scadenza (circa 3 settimane prima della data di discussione affinché i membri delle commissioni di laurea possano visionarle) e accompagnate da un modulo riportante le informazioni generali relative agli autori e al relatore, l'elenco e la descrizione del materiale consegnato, l'autorizzazione (o la non autorizzazione) alla consultazione.

Al fine di ottenere la consegna di elaborati corrispondenti il più possibile ad una struttura redazionale uniforme e di dimensioni ridotte, il CEDAR ha definito alcuni criteri cui i laureandi devono attenersi per la presentazione della tesi. Le disposizioni che, tenendo conto delle diverse tipologie di materiale che caratterizzano le tesi di laurea in architettura, normano l'allestimento sia della classica relazione in volume (obbligo di stampa fronte-retro, un minimo di battute per pagina, indice generale e degli allegati, numerazione delle pagine, tipo di rilegatura) sia del materiale fuori testo (tavole di progetto, diapositive, videocassette ecc.) sono pubblicate in un fascicolo che contiene anche le opzioni per la consegna degli elaborati in formato digitale e il modulo da compilare e consegnare contestualmente alla tesi.

Il fascicolo, che viene periodicamente aggiornato, è disponibile sul sito web del CEDAR <www.cedar.polimi.it/M27.pdf $>$.

L'attività di carattere amministrativo effettuata dal CEDAR in relazione alla raccolta delle tesi prevede quindi:

- supporto informativo (telefonico o in sede) ai laureandi per i chiarimenti sulle modalità di consegna;

- raccolta delle tesi articolata in 2 scadenze (la prima per la tesi su sup-

\footnotetext{
${ }^{3}$ Gli argomenti delle tesi di laurea riguardano gli ambiti più svariati: progettazione architettonica e urbana, pianificazione territoriale, urbanistica, ambientale, design, tecnologia dei materiali, storia dell'arte, restauro, comunicazione visiva ecc.
} 
porto cartaceo, la seconda per la sua versione in formato digitale): il personale verifica che il materiale presentato sia conforme alle disposizioni e che il modulo di accompagnamento sia compilato in tutte le sue parti;

- compilazione di un data-base che raccoglie i dati relativi ai laureandi, ai relatori, al materiale consegnato e alla sua consultabilità e serve ad ottimizzare la gestione dell'archivio (per es. rispondere alle eventuali richieste da parte delle commissioni di laurea nel periodo che intercorre tra la consegna e la discussione; effettuare il controllo delle tesi non consultabili; preparare gli elenchi per la messa in consultazione delle tesi entro un mese dalla loro discussione; contattare i laureandi per eventuali comunicazioni ecc.)

- operazioni di etichettatura delle tesi non consultabili, antitaccheggio, controllo ed eventuale riformattazione dei files contenenti indice e abstract destinati ad essere riversati nel catalogo informatizzato.

\subsection{L'attività di gestione biblioteconomica}

Le tesi raccolte nel corso delle 4 sessioni di laurea di ogni anno accademico inizialmente non furono catalogate (nei primi anni si fece ricorso ad elenchi ordinati per nome del docente relatore), ma già dal 1981 con l'adozione del software INFOBIB creato appositamente per il CEDAR e per la Biblioteca Centrale di Architettura esse furono inserite in un catalogo informatizzato che, evidentemente in modalità testo, offriva possibilità di ricerca semplice (indicazione del codice di un campo seguita dal termine da ricercare) e avanzata (ricerche incrociate mediante operatori booleani).

La catalogazione effettuata secondo lo standard ISBD permetteva quindi non solo la descrizione bibliografica ma un'indicizzazione per titolo, autore, relatore, indirizzo, anno accademico, sessione, parole chiave, ambito geografico e il catalogo del CEDAR poteva essere consultato in sede mediante alcuni terminali.

Il sistema, alla luce degli sviluppi delle tecnologie informatiche risulterebbe ovviamente arcaico ed ostico, soprattutto in fase di catalogazione, ma la sua struttura che prevedeva l'inserimento di codici univoci di campo permise un travaso abbastanza indolore del catalogo verso un sistema più agevole di inserimento, ricerca e visualizzazione dei dati.

Nel 1996 furono infatti adottati i software VIM per la catalogazione e Easyweb per l'OPAC. 
Questo cambiamento di tecnologia rientrava nel progetto di realizzazione dell'OPAC di Ateneo curato dal SIB (Sistema Informativo Bibliotecario) in collaborazione con le biblioteche del Politecnico ${ }^{4}$.

I software, personalizzati a seconda delle caratteristiche dei diversi tipi di materiale, permisero la realizzazione di un catalogo generale consultabile via web mediante maschere di interrogazione diversificate e tipologie di ricerca semplice, per campi, per esperti.

Per quel che concerne le tesi di laurea, l'innovazione portò alla diffusione verso l'esterno di un catalogo contenente già oltre 10.000 documenti (con una conseguente crescita di utenti esterni all'Ateneo) e permise di arricchire l'informazione bibliografica mediante il collegamento all'indice e all'abstract delle tesi. Infatti poiché nuovi software consentono il link e la visualizzazione di files esterni al data-base bibliografico, il CEDAR contestualmente all'avvio del progetto di digitalizzazione delle tesi rese obbligatoria la consegna da parte dei laureandi di un file in formato TXT contenente indice ed abstract da allegare, previa autorizzazione, al catalogo interrogabile anche in Internet ${ }^{5}$.

Il catalogo delle tesi di laurea contenente circa 19.000 record e 4500 indici/ abstract è disponibile all'indirizzo <http://www.biblio.polimi.it/Cataloghi>.

\section{Consultazione}

La consultazione delle tesi di laurea delle Facoltà di Architettura (sia nel formato cartaceo che in quello digitale) avviene esclusivamente presso le due sedi del CEDAR (Leonardo e Bovisa). Le tesi sono quindi escluse dal prestito e in nessun caso ne è consentita la fotocopiatura o la riproduzione con altri mezzi. Le sale di consultazione sono dotate di sistemi di antitaccheggio e tutte le tesi sono protette con strisce magnetiche prima di essere messe in consultazione.

$\mathrm{Al}$ momento della consegna gli autori sottoscrivono sull'apposito modulo l'autorizzazione o la non autorizzazione alla consultazione degli elaborati. In caso di negata autorizzazione gli autori sono tenuti ad indicare il

\footnotetext{
${ }^{4}$ Il software di catalogazione fu adottato dalle biblioteche del Politecnico che non utilizzavano $\mathrm{SBN}$.

${ }^{5}$ Dal momento che l'archivio delle tesi è consultabile solo presso le sedi del CEDAR, la visualizzazione in rete di indice e abstract costituisce un rilevante supporto informativo per individuare con maggior precisione i documenti di possibile interesse soprattutto da parte degli utenti non residenti in Milano.
} 
periodo trascorso il quale la tesi potrà essere resa pubblica. In fase di catalogazione, viene quindi inserita una nota che informa circa la "non consultabilità". Tutte le altre tesi possono essere consultate o su prenotazione (procedura che riguarda le tesi più vecchie) o dietro semplice richiesta accompagnata da un documento di identità. Non c'è un limite numerico per la consultazione, fatto salvo che le tesi vanno richieste una alla volta.

L'analisideidatirelativialle richieste diconsultazionedegliultimi4annidelinea una costante di 22.000 richieste all'anno pari a circa 85-90 tesi al giorno.

Le informazioni sul servizio di consultazione delle tesi sono riassunte all'indirizzo <http://www.cedar.polimi.it/tesi.htm>.

\section{Verso un archivio digitale delle tesi di laurea}

A metà degli anni '90 la riflessione circa il progressivo deteriorarsi del materiale conservato, determinò l'idea di un progetto di digitalizzazione che interessasse almeno una parte dell'archivio.

Un primo progetto (1995-97) si orientò dunque all'individuazione delle tesi più richieste e di quelle di maggior valore scientifico, la cui perdita avrebbe costituito un fattore rilevante nell'impoverimento dell'archivio, e alla digitalizzazione di un campione di 40 tesi effettuata da una ditta esterna mediante scansione delle pagine di testo e delle fotografie delle tavole di progetto allegate, conversione in formato PDF e raggruppamento in un unico file dotato di indice ipertestuale.

Le considerazioni circa la difficoltà incontrata nel contattare gli autori di vecchie tesi per richiedere l'autorizzazione a procedere alla digitalizzazione e la valutazione del rapporto negativo fra la qualità di visualizzazione delle tesi (soprattutto delle tavole di progetto in grande formato che costituiscono parte fondamentale nelle tesi di architettura) e i costi delle procedure di scansione, conversione e creazione dell'indice ipertestuale convinsero il CEDAR a rinunciare (almeno per il momento) all'obiettivo originario di recupero di parte dell'archivio storico.

Al contempo i dati circa l'utilizzo sempre maggiore delle tecnologie informatiche da parte dei laureandi nella realizzazione degli elaborati di tesi, spinsero a ripensare il progetto e a valutare la fattibilità e la definizione di un sistema che, utilizzando il materiale consegnato direttamente dai laureandi in formato digitale, andasse via via a implementare un archivio digitale delle tesi di laurea volto ad agevolare il servizio di ricerca e di consultazione e avente per obiettivo a lunga scadenza la progressiva dismissione della consultazione dell'archivio 
cartaceo (limitata solo ai casi in cui la qualità del materiale su supporti tradizionali si rivelasse effettivamente migliore di quella a video) ${ }^{6}$. Lo sviluppo del nuovo progetto presentava vantaggi non solo per il miglioramento del servizio di consultazione delle tesi, ma anche per la riduzione dei costi e degli spazi occupati e per l'arricchimento formativo dei laureandi (mediante supporto informativo e disponibilità di utilizzo delle strutture informatiche dell'Ateneo) tenuti a presentare gli elaborati secondo determinate modalità.

L'ipotesi prefigurata fu quella della realizzazione di un archivio di files conservati su server, ricercabili mediante una versione personalizzata dell'OPAC esistente (integrato da maschere di registrazione dell'utente) e con accesso limitato ad una rete locale di pc configurati in modo tale da impedire qualsiasi tentativo di copia o di contatto con l'esterno.

Lo studio di fattibilità focalizzò l'analisi sui seguenti punti:

- verifica della possibilità di realizzazione, partendo dal materiale digitale prodotto e consegnato direttamente dai laureandi, di un unico file in formato PDF contenente tutti gli elaborati di tesi (soluzione ritenuta auspicabile in quanto vantaggiosa per l'archiviazione, la conservazione, la protezione e la reperibilità dei documenti) e quindi della possibilità di assumere a standard tale formato;

- quantificazione della dimensione media dei files ottenuti per valutare la quantità di memoria necessaria ad una realizzazione su vasta scala;

- verifica della possibilità di elaborare i files PDF presso il CEDAR per evitare la movimentazione di materiale protetto dal diritto d'autore, velocizzare le procedure di conversione e procedere ad un controllo diretto sulla corrispondenza fra il materiale consegnato in formato cartaceo e quello su supporto digitale.

Come detto in precedenza le tesi di laurea in architettura presentano spesso una composizione variegata: si va dalle tesi costituite da un volume di solo testo a quelle la cui relazione è arricchita da apparati iconografici in bianco e nero e a colori, grafici, tabelle, piccoli disegni, per arrivare alle tesi tipicamente di progetto corredate da diapositive e/o elaborati grafici in formati

\footnotetext{
${ }^{6}$ Il progetto non prevedeva l'abbandono della consegna da parte dei laureandi della tesi in formato cartaceo che viene tuttora considerata come unico documento valido ai fini amministrativi e legali.
} 
che vanno dall'A3 all'A0 a quelle accompagnate da videocassette o il cui fulcro progettuale è un vero e proprio software o un ipertesto multimediale.

Ed è proprio questo aspetto che ha reso la progettazione e la creazione di un archivio digitale decisamente più problematica rispetto alla digitalizzazione di una raccolta di documenti di natura omogenea.

L'analisi delle tipologie di files prodotti dai più diffusi programmi di scrittura e impaginazione, di trattamento delle immagini, di elaborazione di disegni tecnici e la sperimentazione di conversione effettuata dal personale del CEDAR ha portato alla definizione degli standard di realizzazione relativi alla maggioranza delle tesi.

Tenuto conto della rilevanza che riveste per le tesi di laurea in architettura la qualità della riproduzione delle tavole di progetto si è concluso di procedere alla conversione in formato PDF di tutto il materiale che non subisse importanti variazioni di leggibilità a video (files di testo e immagini raster), e di conservare nel formato originario gli elaborati non convertibili come i video digitali (AVI, MOV ecc.) o convertibili ma con elevata perdita di informazione come le immagini vettoriali (DWG, DXF ecc.) prodotte da sistemi CAD e simili.

In definitiva lo standard di realizzazione delle tesi prevede come risultato finale:

- 1 file PDF comprensivo del testo e delle eventuali tavole di progetto (leggibile con Acrobat Reader)

per le tesi composte da files di testo + files raster (per le tavole o le diapositive)

- 1 file PDF con link a files esterni (leggibili con Acrobat Reader + visualizzatori adeguati)

per le tesi composte da files di testo + files vettoriali e/o animazioni.

Tutti ifilesPDF sono successivamente dotatidiun indice ipertestualealfine di agevolare la consultazione del testo e collegare gli eventuali files esterni.

A seguito della delibera del Consiglio di Facoltà che sanciva l'obbligo della consegna delle tesi di laurea anche in formato digitale e come conseguenza delle sperimentazioni descritte sopra, il CEDAR formalizzò per la sessione di laurea dell'aprile 1998 alcune opzioni di consegna allo scopo di ottenere un materiale che fosse sufficientemente raffinato per la successiva elaborazione e conversione in formato PDF senza costituire un eccessivo aggravio di costi per i laureandi.

I risultati delle prime elaborazioni effettuate da parte del CEDAR sul materiale consegnato dai laureandi in formato digitale hanno confermato 
la validità delle scelte riguardo ai formati e della decisione di procedere direttamente al trattamento delle tesi senza rivolgersi a ditte esterne, permettendo inoltre di verificare che la dimensione media di ogni tesi si attesta intorno ai $20 \mathrm{Mb}$ e di prevedere per l'implementazione dell'archivio una necessità di memoria su server oscillante fra i 15 e i $20 \mathrm{~Gb}$ all'anno (dando per scontata, almeno per i primi anni, una realizzazione di tesi non superiore al 50\%).

Con le sessioni di laurea successive il personale del CEDAR addetto al progetto ha provveduto a intensificare il supporto informativo ai laureandi per aumentare la percentuale di tesi consegnate in maniera del tutto corrispondente alle richieste e semplificare quindi le procedure di digitalizzazione diminuendone i tempi di esecuzione e ha implementato l'archivio con l'ausilio di un gruppo di lavoro costituito da studenti collaboratori "150 ore" che ha portato la percentuale delle tesi consultabili direttamente a video dal 20\% dell'anno accademico 1997/98 al 46\% dell'anno accademico 1999/00 (per un totale di 1352 tesi realizzate su 4006 consegnate) ${ }^{7}$.

Come si può notare, il dato sulla realizzazione delle tesi in formato digitale è in costante aumento e sebbene non sia pensabile di conseguire

Riepilogo realizzazione tesi in formato digitale per anno accademico

\begin{tabular}{lccccc} 
& cons & aut & fatte & $\%$ aut & $\%$ tot \\
\hline $97-98$ & 1443 & 1351 & 284 & 21.0 & 19.6 \\
\hline $98-99$ & 1335 & 1122 & 503 & 44.8 & 37.6 \\
\hline $99-00$ & 1228 & 922 & 565 & 61.2 & 46.0 \\
\hline totale & 4006 & 3395 & 1352 & 39.8 & 33.7
\end{tabular}

Legenda: cons=tesi consegnate; aut=tesi i cui autori hanno concesso l'autorizzazione alla consultazione immediata [solo su queste si procede alla conversione e all'archiviazione digitale; le tesi non autorizzate sono destinate, almeno fino a quando l'incremento dei documenti rimarrà così elevato, a non essere recuperate alla scadenza del periodo di non consultabilità]; fatte=tesi realizzate e pronte per la consultazione; \%aut= rapporto tesi realizzate /tesi autorizzate; \%tot=rapporto tesi realizzate/tesi consegnate.

${ }^{7}$ Dal computo sono escluse le tesi dell'anno accademico 2000/01 la cui elaborazione è in fase di completamento. 
nell'immediato futuro l'obiettivo di una totale dismissione della consultazione del cartaceo, non è azzardata l'ipotesi che nel giro di qualche anno si potrà giungere ad avere un archivio digitale contenente l' $80 \%$ delle tesi per le quali è stata concessa l'autorizzazione alla consultazione.

Le tesi realizzate sono masterizzate su cd in duplice copia, una aperta per eventuali successive modifiche e una completamente protetta che viene riversata su server e resa disponibile alla consultazione presso entrambe le sedi del CEDAR da alcune postazioni dedicate.

L'interfaccia di accesso ai documenti è stata affidata inizialmente ad un semplice elenco ipertestuale ordinato per relatore e successivamente ad un motore di ricerca su pagine html statiche (realizzate quindi "manualmente" partendo dai dati sulle tesi contenuti nel catalogo) che ha funzionato discretamente finché l'incremento dell'archivio ha evidenziato come inderogabile il ricorso ad un sistema di ricerca integrato al data-base bibliografico in uso per l'archivio cartaceo che si configura evidentemente come la soluzione ottimale ma la cui realizzazione è legata ai tempi di sviluppo delle politiche di Ateneo per l'automazione delle biblioteche.

Il passaggio ad una versione aggiornata dell'OPAC (o ad una soluzione alternativa) dovrebbe permettere di portare a conclusione il progetto e rendere funzionante a tutti gli effetti il servizio di consultazione delle tesi di laurea in formato digitale permettendo agli utenti di interrogare il catalogo dalle postazioni situate nelle 2 sedi del CEDAR, visualizzare direttamente le tesi archiviate sul server, e richiedere al banco della distribuzione (spostandosi, se necessario, nell'altra sede) solo le tesi di cui non è disponibile la versione digitale. 


\title{
Comunicare l'esperienza: le tesi di laurea nella Biblioteca Centrale di Architettura
}

\author{
Alberta Zanella \\ Biblioteca Centrale di Architettura \\ Sistema bibliotecario \\ Politecnico di Torino
}

"Conoscere e non agire non è ancora conoscere...."

Y. Mishima

\begin{abstract}
Dal 1971 la Biblioteca Centrale di Architettura del Politecnico di Torino scelse di raccogliere tutte le tesi possibili come materiale documentale per renderlo accessibile agli utenti, perché da molto tempo ne era stato intuito il valore. La raccolta delle tesi ebbe origine su impulso dei proff. Roberto Gabetti e Giovanni Brino: dapprima si trattava di una semplice rassegna bibliografica (titolo, autore, relatore, anno accademico), che consentiva agli studenti di orientarsi rispetto agli argomenti già trattati. In seguito alcuni docenti iniziarono a donare le tesi di cui erano relatori. Nel 1984 i laureandi furono invitati a depositare copia della loro tesi, ma soltanto nel 2000 le presidenze di facoltà istituzionalizzarono tale consegna. La mia esperienza trentennale in BCA mi ha portata a considerare le tesi di laurea come il dato più rilevante d'indagine nei campi di ricerca e sperimentazione in architettura, frutto di approfondimento con i relatori e che la facoltà approva scientificamente. Per pubblicizzare le tesi, negli anni 1994-1996, il Laboratorio di Documentazione della Didattica del Progetto, si pose come obiettivo principale l'iniziativa di far circolare la mostra dal titolo "Il Progetto nelle Tesi di Laurea della Facoltà di Architettura di Torino", in modo da far conoscere agli studenti e ai docenti le tesi progettuali svolte nella facoltà, e di farle conoscere nella loro interezza di elaborato conclusivo di un percorso didattico articolato e nella loro consistenza di manufatto unico.
\end{abstract}

Nel lavoro quotidiano nella Biblioteca Centrale di Architettura del Politecnico di Torino si può constatare che le tesi di laurea sono uno strumento di lavoro di abituale consultazione per gli utenti: gli studenti se ne servono per i loro temi di corso, e laureandi e studiosi le utilizzano per approfondire argomenti specifici. Né va trascurato l'interesse che 
alcuni docenti dimostrano nel consultare lavori di altri corsi di laurea, sia per precisare le bibliografie che per attingere da esse alcuni dati di base.

Le tesi possono essere intese, infatti, sia come "prodotto finito", sia come contributo, di diversa estensione e di diverso approfondimento, per lo studio di determinati argomenti.

L'iter formativo dello studente in architettura richiede una preparazione culturale ampia e pluridisciplinare, tale da consentirgli di utilizzare per la progettazione architettonica un adeguato bagaglio di conoscenze, da quelle storiche e urbanistiche a quelle umanistiche, a quelle specificamente tecniche; la tesi rappresenta la conclusione di un lungo lavoro, la prova di una maturazione completa.

Dal 1971 la Biblioteca Centrale di Architettura del Politecnico di Torino scelse di raccogliere tutte le tesi possibili, discusse in facoltà, come materiale documentale per renderlo accessibile agli utenti, perché da molto tempo ne era stato intuito il valore.

La raccolta delle tesi ebbe origine su impulso dei proff. Roberto Gabetti e Giovanni Brino: dapprima si trattava di una semplice rassegna bibliografica (titolo, autore, relatore, anno accademico), che consentiva agli studenti di orientarsi rispetto agli argomenti già trattati. In seguito alcuni docenti iniziarono a donare le tesi di cui erano relatori. Nel 1984 i laureandi furono invitati dall'allora preside della facoltà prof. Lorenzo Matteoli a depositare copia della tesi, dando così l'avvio alla costituzione di un Settore importante per la biblioteca, ma soltanto nel 2000 le presidenze di facoltà istituzionalizzarono tale consegna.

La mia esperienza trentennale in BCA mi ha portata a considerare le tesi di laurea come il dato più rilevante d'indagine nei campi di ricerca $\mathrm{e}$ sperimentazione in architettura, frutto di approfondimento con i relatori e che la facoltà approva scientificamente.

Sfogliando il catalogo, si può notare che gli interessi di studenti e docenti rispondono a richieste pluralistiche, toccando tutte le aree culturali.

Con il passaggio tra vecchio e nuovo ordinamento sino ai nuovi corsi di laurea in architettura, le tesi rispecchiano l'iter diversificato che ha finora caratterizzato la preparazione degli architetti; si rileva inoltre che la maggior parte delle tesi ha un forte contenuto progettuale e che investe campi differenziati, che vanno dal restauro al tecnico-strutturale, all'urbanistico al sociologico, all'impiantistico, all'estimativo allo storico-architettonico e al design.

Dal 1973 il metodo di schedatura adottato rifletteva esperienze internazionali; l'obiettivo per facilitarne la consultazione aveva portato a ordi- 
nare il catalogo a schede secondo le seguenti chiavi di accesso: Materia, Autore, Relatore, Parole-chiave). In parallelo c'era un catalogo a schede e a stampa, in cui le tesi erano ordinate secondo un numero progressivo d'ingresso, che ne indicava anche la collocazione.

Sin dall'inizio furono collocate a scaffale aperto e ordinate secondo numero progressivo di ingresso.

Nel 1986 nasce il Sistema Bibliotecario del Politecnico, con il compito di coordinare le biblioteche dell'Ateneo: le Biblioteche Centrali di Ingegneria e Architettura, le biblioteche delle Sedi decentrate, e numerose Biblioteche di settore e dipartimento. La suddivisione delle biblioteche di settore, che possono fare capo a uno o più Dipartimenti, rispecchia una ripartizione in aree culturali e scientifiche.

L'elemento di coesione è l'informatizzazione dei cataloghi: con Minisis si crea un database specifico per le tesi, che nel frattempo vengono raccolte anche da alcune biblioteche di settore.

La scheda catalografica informatizzata presentava campi comuni a tutte le biblioteche.

I campi erano:

1-Numero progressivo d'ingresso,

2-Sigla del Corso

3-Sigla biblioteca

4-Candidato (rip.),

5-Titolo

6-Relatore (rip.)

7-Descrizione fisica

8-Note

9-Parole-chiave

10-Data.sigla del catalogatore+note.

Dal 1994 è possibile interrogare gli archivi delle registrazioni catalografiche attraverso un'interfaccia WEB <http:/ /:wwwbiblio.polito.it $>$

Con l'avvio del nuovo ordinamento dal 1997 scompare per la Facoltà di Architettura il campo Sigla del Corso (materia), per lasciare posto al campo Area della tesi.

Nel 2001 le tesi presenti nell'archivio Tes in Minisis sono state migrate in Aleph seguendo il template predisposto dal gruppo di lavoro "Aleph tesi”.

Il template è il seguente: 


\begin{tabular}{ll} 
DB & PTO01 \\
SYSID & \multicolumn{1}{c}{ TH } \\
FMT & TH \\
LDR & nam-22-450- \\
& \multicolumn{1}{c}{$100 \quad$ a-d $101 \quad$ ita } \\
& titolo e resp. \\
2001 & pubblicazione \\
210 & descrizione fisica \\
215 & nota: Tesi di laurea \\
328 & autore persona \\
7001 & relatore \\
7091 & IT SBPT RICA Unimarc \\
8010 &
\end{tabular}

Nonostante la cronica carenza di spazi, questo settore offre un sempre più ampio servizio in termini di consultabilità del materiale anche attraverso verifiche inventariali integrative.

Infatti nel luglio 1999 si sono colmate delle lacune con un lavoro di recupero di tesi pregresse, giacenti presso la Segreteria della Facoltà, e ancor oggi alcuni docenti lasciano le copie in originale di cui sono relatori, che vanno a sostituire la copia di deposito, in quanto più complete.

Attualmente le tesi sono 9408 (aprile 2002), per un totale di 590 metri lineari.

Il numero di tesi prodotte per anno presso le facoltà è dell' ordine di circa 450 unità; risultano a disposizione per i futuri volumi 126 metri lineari.

Il personale impiegato per il trattamento di questo materiale è il gruppo schedatura, che se ne occupa a tempo pieno nel periodo in cui pervengono dalle Presidenze. L'operazione comprende: attribuzione del numero progressivo, schedatura, timbratura, magnetizzazione e cartellinatura. Inoltre su ogni allegato vengono riportati numero di ingresso, timbro e banda magnetica.

Per tutelare il diritto d'autore il Regolamento della BCA permette solo la fotocopiatura dell'indice e della bibliografia, e ne vieta il prestito.

Molte tesi di architettura sono assegnate su temi locali, e a volte risultano essere l'unico materiale esistente in biblioteca su quegli argomenti.

Altre volte le tesi sono appetibili perché trattano temi di attualità immediata.

Talvolta le stesse vengono utilizzate dai docenti per alcune lezioni, in modo che gli studenti possano fare riferimento a queste quando preparano gli esami. 
Come si può capire i furti sono molto diffusi: vengono asportati sia i volumi rilegati che parti (per esempio le tavole di progetto).

Per prevenire i furti si adottano un maggior controllo da parte del personale e la magnetizzazione con bande magnetiche, applicate anche alle tavole allegate.

Si riscontra che il formato e i criteri grafici delle tesi sono di fantasia, e questo produce problemi alla catalogazione (dati mancanti nel frontespizio, nell'indice...) e di collocazione fisica delle medesime.

Per ovviare a questi problemi si è richiesto alle Presidenze di far adottare ai tesisti la Norma Italiana UNI ISO 7144, riferita alla presentazione delle tesi, ma ad oggi non si è avuto nessun riscontro. La biblioteca fa opera di sensibilizzazione presso i docenti, affinché questi criteri siano comunque adottati, anche se non obbligatori.

Nel 1995, per occupare minor spazio, si è sondata la possibilità di riversarle su supporti diversi da quello cartaceo, quali microfiche, microfilm e Cd-rom, tanto più che già dal 1991 il Centro Interdipartimentale Servizi Didattici Architettura aveva incominciato a sperimentare tesi multimediali salvate su supporti digitali (Cd-rom).

Nel 1997 il direttore prof. Giovanni Ghione ha approvato che il progetto di archiviazione delle tesi fosse interamente finanziato dal Sistema Bibliotecario. Il supporto più interessante è risultato il cd-rom; lo studente avrebbe dovuto consegnare i documenti in formato WORD (già diventato lo standard di fatto), lo stesso, era disponibile in multipiattaforma presso i laboratori informatici, ed era immediatamente traducibile in formato HTML tramite versione 7.0 di WORD.

I formati grafici per i disegni tecnici erano in DVG e per le tavole in PCX, trasformato poi in GIF per la consultazione online.

Per quanto riguardava la consultazione in rete, i Servizi Informatici di Facoltà avrebbero dovuto farsi carico di inserire la tesi in linea full-text.

La parte più interessante era l'opzione da progettare e studiare ed infine realizzare, per la costruzione di un motore di ricerca per Item che analizzasse tutte le tesi inserite online.

Questo progetto non è stato approvato dal Consiglio di Facoltà perché presentava molteplici problemi: per esempio molti laureandi sostituiscono ancor oggi, in parte o completamente, la copia già consegnata con l'ultima stesura della tesi, completata a volte il giorno dopo la discussione.

Lo studio di archiviazione delle tesi di laurea in formato elettronico riguardava anche il pregresso e lo studio di fattibilità, che ha prodotto due prototipi, era stato affidato ad un provider esterno: si è mostrata la capacità di tradurre tesi dal formato cartaceo o parzialmente elettronico 
in formato HTML, visibile, in forma multimediale, attraverso comuni browser Internet.

A partire dalla sessione di laurea del luglio 1996 le tesi ritenute dalla Commissione di laurea più meritevoli sono pubblicate in forma di estratto (4.000 battute più eventuali illustrazioni e tavole) sulla pagina web $<$ http:/ /obelix.polito.it>

Inoltre a Torino si costituisce, a partire dal 1998, un gruppo di studio sulla letteratura grigia e materiale minore, a cui tuttora partecipo per il Sistema Bibliotecario.

Si tratta di un confronto tra enti diversi ( Biblioteca Internazionale di Cinema e Fotografia "Mario Gromo", Biblioteca Musei Civici Galleria d'Arte Moderna, Ufficio Documentazione CCIAA di Torino, Biblioteca del Teatro Stabile Torino, ArtLab...), indirizzati alla verifica di uno standard descrittivo di quel tipo di materiale (di cui fanno parte anche le tesi).

Parallelamente, il LADIPRO (Laboratorio di Documentazione della Didattica del Progetto) sin dal 1994 dava avvio alla propria attività con una mostra di tesi di laurea progettuali della Facoltà di Architettura segnalate dalla commissioni di laurea. La documentazione relativa alle mostre di cadenza annuale è pubblicata nei cataloghi che presentano lo stesso titolo Il progetto nelle tesi di laurea della Facoltà di Architettura del Politecnico di Torino, Torino, Celid..

La commissione preposta all'organizzazione delle mostre sceglie le tesi da esporre basandosi sulle copie conservate in biblioteca. In questo contesto la collezione diventa il punto di riferimento per il progetto espositivo.

Nel 2000 nasce Webthesis (le tesi in rete): il progetto promosso dalla Biblioteca Centrale di Architettura in collaborazione con il Laboratorio Produzioni Ipermediali HYPARC del CISDA si è posto l'obiettivo di acquisire i dati significativi relativi alle tesi di laurea.

Concludendo per il futuro, secondo un mio peraltro personale giudizio, sarebbe auspicabile che la raccolta delle tesi di laurea, che presenta sempre un alto coefficiente di interesse, trovasse risorse finanziarie e spazi adeguati per conservare integralmente il materiale pregresso, per documentare il presente e valorizzare il passato con l'uso delle nuove tecnologie.

Molto c'è da fare, anche rispetto al trattamento bibliografico: ad esempio, le citazioni bibliografiche delle tesi di laurea attualmente adottate mancano di uniformità e non sono esaustive (mancano le indicazione dei relatori, correlatori... vedi ISO 7144 - Documentation-Presentation of theses and similar documents). La citazione bibliografica di una tesi dovrebbe contenere i seguenti dati: 
Autore/i della tesi [Cognome, Nome (per esteso)], Titolo e sottotitolo, anno, Tipologia della tesi (tesi di diploma, tesi di laurea, tesi di master...), Relatore/i, Correlatore/i, Città, Università, e indicazioni sul centro di documentazione che conserva la tesi. 



\title{
Il progetto Tesi-Meritorie delle Facoltà di Architettura del Politecnico di Torino
}

\author{
Evandro Costa \\ Centro Interdipartimentale Servizi Didattici di Architettura \\ Politecnico di Torino
}

Dal 1996 è attivo presso il CISDA del Politecnico di Torino un pogetto volto alla valorizzazione di tesi segnalatesi in corso di discussione, con l'immissione in formato digitale in Web per una fruizione allargata ad una utenza specialistica.

Il CISDA (Centro Interdipartimentale Servizi Didattici di Architettura) nel 1995 ha proposto alla Facoltà di Architettura la pubblicazione sul WEB delle tesi giudicate "particolarmente meritevoli" dalle Commissione di Laurea; i servizi HypArc (Hypertext for Architecture, hyparc@polito.it) e SLA (Servizio linguistico di Architettura) si sono fatti carico della progettazione della pagina tipo, dell'impianto di navigazione e consultazione, della preparazione dei materiali da pubblicare e della revisione della traduzione inglese.

Il progetto, avviato nel 1995, è presente nel WEB dal Luglio 1996.

Il progetto tesi meritorie persegue i seguenti obiettivi :

- permettere alle commissioni di Laurea di sottolineare la qualità della ricerca espressa da alcune tesi di laurea; l'alto livello di ufficialità della "meritorietà" è proprio espresso dalla commissione di laurea, che seguendo criteri di giudizio sulla base della maggioranza dei membri, raggiunge il giudizio di valutazione;

- rendere pubblici gli abstract delle tesi meritorie tramite il WEB, con un limite di 4000 caratteri e tre immagini;

- rendere disponibili gli abstract in lingua italiana e inglese, e concedendo la possibilità di inserire ulteriori versioni (significativo l'esempio di una tesi in versione swaili, a seguito del lavoro di ricerca effettuato in Nigeria); 
- promuovere tramitela posta elettronicacontattitrailmondoesterno (settore pubblico, universitario, privato) e i neolaureati. Effettivamente dal feedback riscontrato dal servizio di gestione risulta che le Tesi vengono "viste" e spesso creano "link virtuosi" tra il mondo esterno e l'università.

In particolare, le operazioni finora svolte sono state le seguenti:

- acquisizione dei testi dell'abstract in formato MS-Word o txt (massimo 4000 caratteri) e delle immagini (massimo n.3 ) in formato digitale; l'abstract e le immagini digitali possono essere inviate al servizio via posta elettronica; risulta essere quest'ultimo il metodo più utilizzato;

- controllo linguistico della versione inglese;

- eventuale scansione ed elaborazione delle immagini (nel caso di foto su supporto cartaceo);

- formattazione del testo e delle immagini nella pagina con l'inserimento del testalino uniformato per tutte le tesi (Facoltà, Corso di Laurea, titolo della tesi, nome tesista, relatore, correlatore, e-mail del tesista);

- invio dei materiali al Centro Servizi Informatici C.E.S.I.T. per la pubblicazione sul sito della Facoltà.

Il sistema informativo sta migrando da una versione statica costituita da pagine elaborate in linguaggio html, ad una versione in formato PDF (Adobe Acrobat) in cui è prevista una ricerca per parole chiave (versione fine 2002). I dati vengono quindi inseriti in un Data-Base che permette la successiva ricerca dinamica per parole chiave.

La sensibilizzazione della docenza verso tale concessione di meritorietà è andata aumentando negli anni, ora tale concessione è diventata un punto di merito che viene apprezzato e fortemente discusso in sede di valutazione finale, e viene concesso esclusivamente a lavori di ricerca particolarmente apprezzati dalla commissione di laurea stessa.

La "dignità di stampa" è quindi migrata da un sistema costoso e poco diffuso, ovvero quello tradizionale cartaceo, ad un sistema che soddisfa esigenze di "visibilità", di "pubblicizzazione" e di "contatto diretto" dei lavori di ricerca dei laureandi, di cui si possa considerare scontato l'interesse.

Alcuni aspetti credo debbano essere sottolineati, in particolare l'interesse riscontrato per tale iniziativa. Dalle e-mail ricevute dal servizio HypArc risulta che:

- il sito riscuote un forte interesse da parte degli studenti che spesso si trovano in una analoga fase di ricerca; 
- Il sito è visitato da Amministratori Pubblici interessati a studi sulle realtà locali, a cui spesso seguono contatti diretti con il tesista per collaborazioni;

- Il sito è visitato da aziende private che ricercano personale specializzato in ambiti particolari (significative alcune e-mail rivolte a laureandi specializzatisi in riciclo dei materiali e in design di oggetti di uso particolare);

- L'accesso alle versioni in lingua inglese è apprezzabile, quindi si presume che il sito venga visto anche dall'estero;

- La pubblicazione ufficiale sul WEB costituisce un punto di merito consistente per il curriculum del laureato, peraltro pubblico e raggiungibile facilmente tramite un URL personale.

- Il CISDA continua a svolgere il servizio fin qui svolto, cioè la predisposizione dei materiali e il loro upload: l'attuale responsabile del servizio è Evandro Costa (evandro.costa@polito.it). La presenza di un unico incaricato per le due facoltà assicura una omogeneità nel trattamento dei dati.

In totale tesi pubblicate sino ad oggi (da luglio 1996 a maggio 2002) sono 377:

1996: n. 40

1997: n. 58

1998: n. 72

1999: n. 64

2000: n. 55

2001: n. 79

2002: n. 9

Gli accessi sono stimati a circa 10.000 l'anno, di cui ben 2500 dall'estero, esclusivamente per la versione inglese. 



\title{
Il progetto WebThesis
}

\author{
Daniela Ferrero \\ Biblioteca centrale di Architettura \\ Sistema bibliotecario \\ Politecnico di Torino
}

\begin{abstract}
Il progetto WebThesis tende a mettere a disposizione della consultazione esterna l'archivio delle tesi con il più ampio corredo informativo possibile. L'obiettivo è di mettere in rete tutte le tesi in formato digitale, a regime, anche se attualmente le linee di intervento operano per fornire uno schema sintetico dei contenuti delle tesi.
\end{abstract}

Il progetto ha nella sua versione completa un titolo e un sottotitolo: WebThesis (le tesi in rete). Il sottotitolo, inserito per rendere esplicito soprattutto agli studenti, il contenuto del progetto, può apparire troppo ambizioso. In realtà, se per un verso pecca per eccesso, perché non si tratta della versione integrale delle tesi on-line, per l'altro verso non esplicita completamente lo sforzo di sintesi mirato a fornire accessi facilitati alle informazioni contenute nelle tesi. La precisazione contenuta nel sottotitolo si è comunque rivelata una scelta efficace per la comunicazione e per la promozione dell'iniziativa.

Il progetto si inserisce in un quadro di riferimento che ha visto e che vede la Biblioteca Centrale di Architettura del Politecnico di Torino dedicare una particolare attenzione alle tesi di laurea. Le esperienze consolidate della Biblioteca, illustrate dall'arch. Zanella, e la più recente iniziativa delle "tesi meritorie", illustrata dall'arch. Costa, hanno rappresentato i precedenti di una linea di lavoro orientata a valorizzare e a mettere a disposizione della consultazione esterna l'archivio delle tesi con il più ampio corredo informativo possibile. Questa precisa volontà era accompagnata dalla consapevolezza di una situazione locale non ancora matura per prevedere un archivio completo della tesi in formato elettronico ${ }^{1}$. Non

\footnotetext{
${ }^{1}$ Per raggiungere tale obiettivo dovranno essere garantiti agli studenti i necessari supporti tecnici e di servizi.
} 
si esclude in futuro di poter raggiungere tale obiettivo, ma se non è credibile nell'immediato, non è in contrasto con il progetto avviato, anzi, in un'auspicabile situazione di regime (WebThesis per tutte le tesi e versione digitale delle stesse) i due prodotti costituiranno il giusto completamento l'uno dell'altro. WebThesis permetterà, attraverso la ricerca su indicatori sintetici, l'individuazione della tesi che interessa, che potrà essere consultata in rete, anziché sull'originale cartaceo disponibile presso la biblioteca, come avviene attualmente.

Attualmente ${ }^{2}$ è disponibile un catalogo informatizzato delle tesi con una possibilità limitata di accessi alla ricerca. Sono possibili ricerche per le seguenti voci: Candidato, Relatore, Parole del titolo, Parole chiave ${ }^{3}$, oppure per sessione di discussione della tesi. Le opzioni fornite da WebThesis sono di ben altra portata.

Il riconoscimento dell'importanza del patrimonio informativo e documentario contenuto nelle tesi, dato ormai consolidato e condiviso, è stato infatti di stimolo per migliorare la quantità e la qualità delle informazioni messe a disposizione con la consultazione dei cataloghi o con repertori ad essi paralleli, che si riferiscono ad una tipologia di materiale dai caratteri di unicità dell'esemplare.

Si è però riscontrato come spesso a fianco di ricchi contenuti siano rilevabili vistose lacune nella struttura metodologica di restituzione finale dei contenuti. Un esempio di queste carenze è rappresentato dalla mancanza di contenuto informativo negli indici delle tavole. È infatti frequente la semplice indicazione della successione numerica delle tavole (ta. 1, 2, ecc.) senza nessun altra indicazione che è invece presente nell'intestazione di ogni singola tavola.

Si è quindi pensato ad un progetto che potesse recuperare, in termini di contenuti informativi, i principali elementi riepilogativi delle tesi, con una previsione di consultabilità esterna.

Da queste premesse è stato elaborato un progetto che ha ottenuto un finanziamento nell'ambito dei "Progetti per il miglioramento della qualità della didattica” della I e II Facoltà di Architettura del Politecnico di Torino. Il progetto, promosso in collaborazione dalla Biblioteca Centrale di Architettura del Sistema Bibliotecario e dal Laboratorio Produzioni Ipermediali HYPARC del CISDA del Politecnico di Torino, è stato finanziato nel mese di giugno 2000.

\footnotetext{
${ }^{2}$ Cfr. le indicazioni fornite dall'intervento dell'arch. Zanella.

${ }^{3} \mathrm{Si}$ tratta di termini, inseriti dai bibliotecari in fase di catalogazione per aumentare il contenuto informativo della scheda catalografica. Sono indicatori relativi principalmente a indicazioni geografiche, cronologiche, tipologiche e tecnologiche
} 
Ottenuto il finanziamento si è passati dall'elaborazione concettuale alla fase tecnico-operativa ${ }^{4}$.

Le informazioni che costituiscono il data base, interrogabile in rete, sono state organizzate in due blocchi distinti che evidenziano la differenza delle informazioni contenute. Il primo blocco contiene le informazioni relative all'identificazione della tesi, utili anche per la sua reperibilità, nel secondo blocco sono contenute le informazioni sintetiche relative ai contenuti della tesi, comprese le indicazioni sul materiale allegato al testo. Le voci in cui sono articolate le due sezioni sono le seguenti:

Identificativi della tesi:

- Cognome, Nome

- Matricola

- Corso di Laurea, Facoltà

- E-mail

- Titolo della tesi

- Relatore / Correlatore

- A.A. sessione

Contenuto della tesi:

- Indice della tesi

- Indice delle tavole (con didascalie)

- Indice dei materiali allegati (iconografia, documenti d'archivio)

- Abstract

- Bibliografia

- Parole chiave

Caratteristica di WebThesis è che l'immissione dei dati avviene a cura dello studente, con un ovvio alleggerimento del lavoro di data-entry a carico delle strutture bibliotecarie, onere che da sempre ha frenato iniziative di analogo impegno. Per la compilazione delle voci sono fornite al laureando istruzioni specifiche. Queste istruzioni, disponibili in rete, sono sia di tipo tecnico (es. formato dei files) sia di natura esplicativa sul significato delle voci (es. descrizione dei materiali allegati).

È evidente come le voci che compongono la sezione relativa al conte-

\footnotetext{
${ }^{4}$ Per un approfondimento specifico cfr. l'intervento dell'ing. Tealdi e Venezia.
} 
nuto delle tesi costituiscono l'aspetto innovativo e dall'elevato potenziale informativo per svolgere ricerche su questo tipo di materiale caratterizzato dall'unicità degli esemplari, come precedentemente richiamato.

Il progetto è attualmente in fase di sperimentazione e si sta operando per la definitiva messa a punto degli aspetti tecnici e funzionali della base dati. Al termine di questa fase sperimentale e di test è previsto il passaggio dall'attuale adesione volontaria da parte degli studenti (che ha comunque ottenuto una buona percentuale di adesioni) ad una partecipazione obbligata, concordata con le Presidenze di Facoltà. L'immissione dei dati in WebThesis diventerà in futuro vincolante per l'ammissione alla discussione della tesi.

È necessario a questo punto fare alcune precisazioni. Il progetto si è sviluppato come raccolta del patrimonio informativo delle tesi che concludevano il corso di studi di durata quinquennale. Da subito è stato deciso di non effettuare il recupero del pregresso, troppo oneroso in termini di energie, perché il data-entry sarebbe stato in questo caso completamente a carico della Biblioteca. Contemporaneamente all'avvio del progetto, si è passati al nuovo modello formativo con l'avvio dei corsi di studi triennali seguiti dal biennio delle lauree specialistiche. Al termine del triennio è comunque prevista per lo studente l'elaborazione di una prova finale, ma i caratteri di questa prova finale, che si è andata definendo come una riflessione critica dell'esperienza triennale, non contengono quell'intreccio fra didattica e ricerca che sta alla base dell'interesse per le tesi di laurea. Da questa valutazione è derivata la scelta di limitare il progetto alle lauree quinquennali, fino al loro esaurimento, per proseguirlo poi con le future lauree specialistiche dei diversi corsi di studi attivati presso la I e la II facoltà di Architettura.

Il progetto si propone anche come modello per possibili utilizzi da parte di altre strutture bibliotecarie dell'Ateneo, depositarie di tesi di laurea per esempio la Biblioteca Centrale di Ingegneria e alcune biblioteche di settore o di sedi decentrate. 


\title{
Progetto WebThesis: aspetti tecnici
}

\author{
Paolo Tealdi, Emanuele Venezia \\ Servizi centrali informatici e bibliotecari \\ Sistema Bibliotecario \\ Politecnico di Torino
}

Si presenta la struttura del progetto WebThesis, dallo spiccato carattere di collegamento tra il mondo dell'università e quello del mondo del lavoro.

\section{Obiettivi}

WebThesis nasce con l'intento di fornire un contatto tra il mondo del lavoro e i neolaureati. L'idea è quella di fornire alle aziende, oltre al catalogo delle tesi sviluppate nell'Ateneo, delle schede informative completate da un abstract, a cura del candidato stesso, e da informazioni aggiuntive per contattare direttamente lo studente.

\section{Procedure}

Il funzionamento del sistema può essere descritto con il seguente diagramma di flusso di dati, in cui sono presenti tre agenti esterni, il server Aleph e le funzioni che costituiscono il progetto WebThesis.

Il laureando, attraverso un'interfaccia web, inserisce i propri dati di base (nome, cognome, indirizzo di posta elettronica, corso di laurea di appartenenza, sessione di laurea), il titolo della tesi, il relatore e gli eventuali correlatori, un abstract, i titoli dei capitoli e delle tavole, la bibliografia e un elenco di parole chiave per la ricerca (in parte come soggetti liberi, in parte scegliendoli da un elenco).

Il sistema informativo elabora le informazioni inserite e prepara un riepilogo (un documento pdf) che spedisce al laureando, e un record in formato Unimarc pronto per l'inserimento nel catalogo collettivo. 


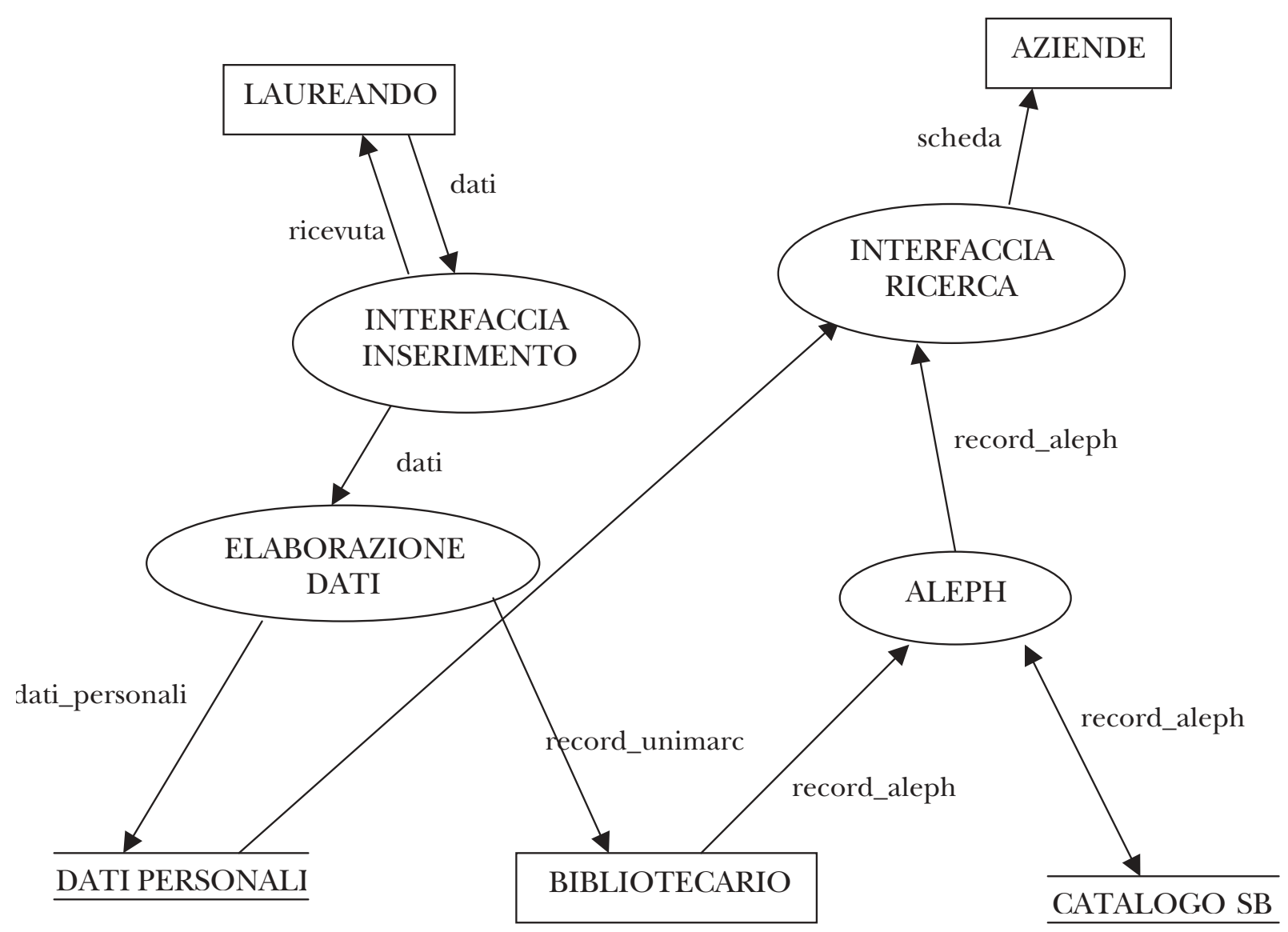

dati $=\{$ nome, cognome, email, titolo_tesi, abstract, capitoli, tavole, illustrazioni $\}$ ricevuta $=\{$ riepilogo_dati_inseriti $\}$ scheda $=\{$ pdf_laureato_tesi $\}$ dati_scheda $=$ \{dati_personali, record_aleph $\}$ dati_personali $=\{$ nome , cognome, email $\}$ record_unimarc $=$ \{record_formato_unimarc $\}$ record_aleph $=$ \{record_formato_aleph $\}$

Il bibliotecario controlla che il record prodotto automaticamente dal sistema sia corretto secondo le norme di catalogazione ed effettua l'inserimento nel database attraverso la GUI di Aleph. Da questo momento il record è visibile nell'OPAC.

Alle aziende si fornisce un'interfaccia web che consente loro di interrogare il catalogo delle tesi, direttamente su Aleph, secondo diversi criteri (per nome del candidato, per titolo della tesi, per parole chiave...), ma in aggiunta hanno la possibilità di scaricare una scheda riepilogativa che contiene anche i dati non presenti in catalogo (principalmente l'indirizzo di posta elettronica) e quindi contattare direttamente il candidato prescelto.

Le funzioni che compongono il sistema sono: due diverse interfacce utente, una per gli studenti, per l'inserimento, e una per la ricerca da 
parte delle aziende; algoritmi batch per la conversione dei dati e la generazione delle schede informative e del record Unimarc e infine l'interazione e comunicazione con il server Aleph. Inoltre l'interfaccia a disposizione dei laureandi deve gestire l'autenticazione, la possibilità di inserire i dati in diversi momenti, eventualmente modificarli, e inviarli solo quando la scheda è completa.

\section{Architettura dei sistemi}

Il tutto è stato realizzato configurando un server web Apache su piattaforma FreeBSD, cui abbiamo affiancato programmi perl con i moduli MARC, ASP e CGI. Questa struttura deve interagire con il server Aleph, che si appoggia ad un DBMS Oracle, entrambi installati su una Sun con sistema operativo Solaris.

Il vantaggio di questa scelta è stato quello di aver potuto sviluppare tutto in loco, con una minima spesa, e una buona affidabilità, poiché il server Apache e il sistema operativo FreeBSD sono gratuiti e molto conosciuti per la stabilità e la sicurezza.

Inoltre la possibilità di sviluppare all'interno la comunicazione tra questa nuova struttura e il sistema preesistente Aleph, ha permesso di evitare la ripetizione di dati, con conseguente rischio di incoerenza tra gruppi di informazioni.

Infatti una buona parte delle informazioni necessarie per il progetto sono la descrizione bibliografica della tesi, che va inserita nel database principale delle tesi dell'Ateneo, mentre sarebbe stata una forzatura inserire il resto delle informazioni nello stesso.

Diventava dunque necessario separare dal database principale le informazioni personali dell'utente, non previste dagli standard di catalogazione. E non esistendo un'applicazione che permettesse di integrare i due gruppi di dati in una singola interfaccia, l'unica alternativa alla soluzione poi scelta sarebbe stata quella di ripetere tutte le informazioni in un database ad hoc. 



\title{
La gestione delle tesi in formato elettronico allo IUAV
}

\author{
Pierre Piccotti \\ Servizi Bibliografici e Documentali \\ Servizi Informativi Bibliografici e Documentali \\ IUAV - Venezia
}

\begin{abstract}
Si presenta lo stato dell'arte della disponibilità delle tesi in formato elettronico allo IUAV-Venezia, attraverso la trasformazione da formato analogico tradizionale a formato digitale, con ampio recupero, benchè per settori, a partire dal 1988, in sostituzione della precedente microfilmatura.
\end{abstract}

\section{I dati originali}

La prima considerazione da farsi è: a cosa facciamo riferimento?

Infatti, purtroppo, solo da un anno riusciamo ad avere tesi in formato completo; prima, le versioni forniteci non necessariamente erano quelle effettivamente presentate al momento della laurea ${ }^{1}$.

Le tesi che ci vengono fornite sono ancora generalmente in formato cartaceo, salvo eventuali allegati (cd-rom, VHS, etc.). Solo recentemente è iniziata la fornitura di tesi in originale in formato digitale, purtroppo non in maniera organica né controllata. Di fatto soggetta al libero arbitrio di docenti e laureandi.

\section{Patrimonio}

Ovviamente non sono ancora disponibili le tesi relative al nuovo ordinamento. Al momento il patrimonio consta in:

\footnotetext{
${ }^{1}$ Questa considerazione era valida al momento della presentazione dell'intervento. Oggi ci ritroviamo nella medesima situazione.
} 
Tesi di laurea:

- corso di laurea in architettura: tutte dal 1983 a oggi (degli anni precedenti sono possedute solo quelle del Dipartimento di Storia dell'Architettura DSA, a partire dal 1968, con lacune)

- corso di laurea in Pianificazione: dal 1976 a oggi (con lacune).

Tesi di diploma:

- Tutte.

Tesi di dottorato:

- Possedute ma reperibili sono nel catalogo SBN e trattate similmente alle monografie.

\section{Utilizzo}

Principalmente ci risulta che siano consultate soprattutto per il lavoro di documentazione e di ricerca: le fonti utilizzate, le bibliografie, i documenti d'archivio, etc.

Non va escluso il tentativo di copiare tesi altrui...

\section{Software utilizzati}

Catalogazione:

Dal 1983 al 1998 utilizzavamo il software TinLib (IME di Londra, distribuito da IFNET di Firenze). Nel 1999, avendo adottato EasyCat (Nexus SpA di Firenze) per la catalogazione di tutti i materiali "non SBN", abbiamo convertito i dati dei database preesistenti per il nuovo software. Relativamente alle Tesi non abbiamo avuto grossi problemi di conversione. Dal marzo 1999 tutte le tesi vengono regolarmente catalogate con EasyCat.

\section{OPAC:}

In un primo tempo abbiamo realizzato e utilizzato l'OPAC a carattere EasyPac, accessibile via telnet; questo a partire dal 1993. Successivamente, 
a partire dal 1995, avendo realizzato con la Nexus SpA il software EasyWeb (OPAC multimediale in ambiente Web), tutti i cataloghi, tesi comprese, sono accessibili via Web.

\section{Scheda tipo}

Tutti i dati sono visibili direttamente dalla pagina Web del dell'OPAC IUAV: <http://opac.iuav.it >.

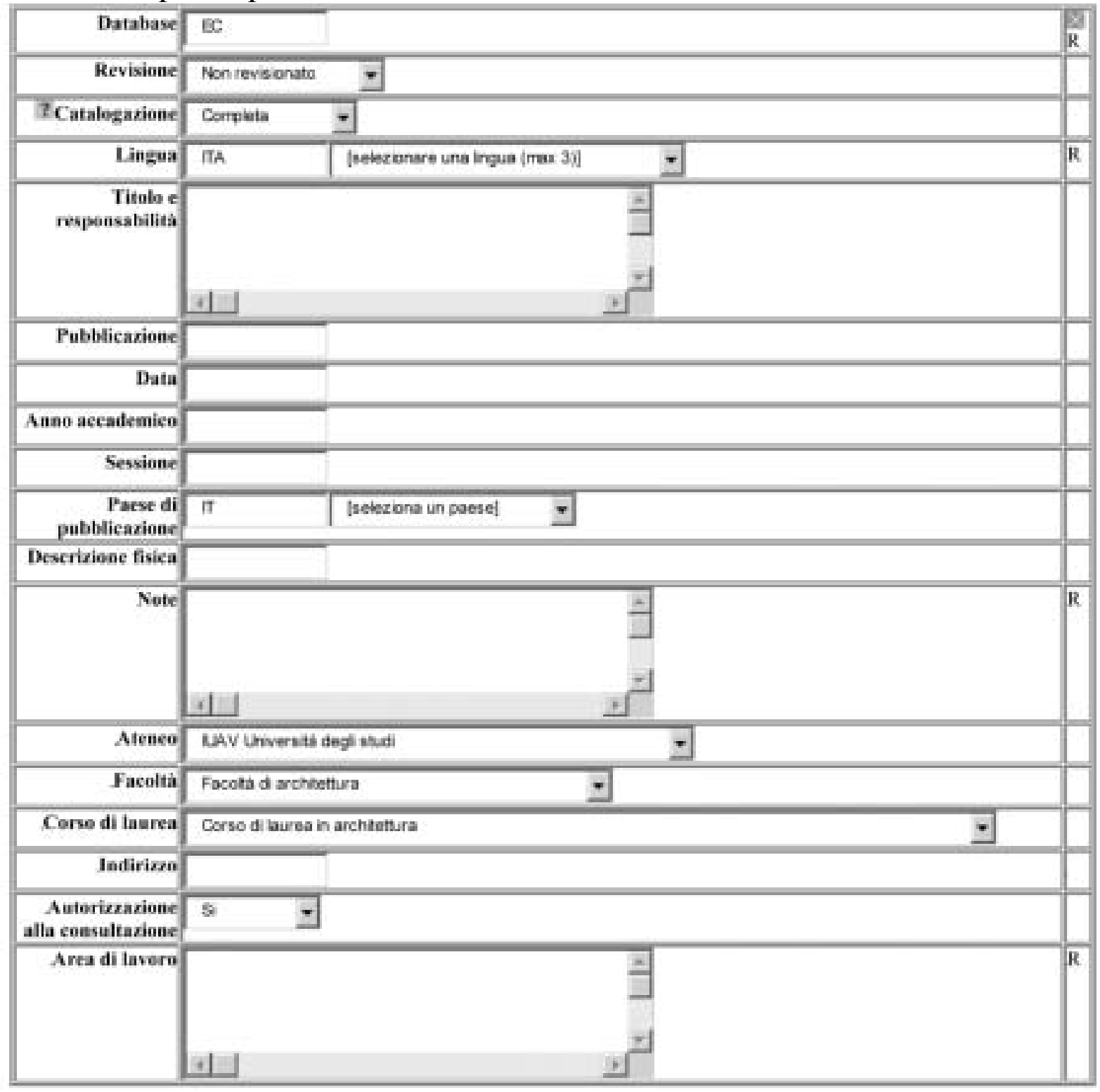

Dove " $\mathrm{R}$ " indica che il campo è ripetibile. 
I seguenti campi si appoggiano invece ad authority file:

- Autore livello 1 e 2

- Relatore / correlatore.

In OPAC ritroviamo la seguente struttura:

\begin{tabular}{|c|c|c|}
\hline Codice campo & Contenuto & Modalità di ricerca \\
\hline $\mathbf{K W}=$ & Termini presenti in tutti campi & Parole \\
\hline $\mathbf{A} \mathbf{U}=$ & Autore & Parole \\
\hline $\mathbf{R L}=$ & Relatore & Parole \\
\hline $\mathbf{T I}=$ & Titoli & Parole \\
\hline $\mathrm{PA}=$ & Paese di pubblicazione & Stringa ISO \\
\hline $\mathrm{PP}=$ & Paese di pubblicazione & Parole ISO \\
\hline SO= & Soggetto & Parole \\
\hline $\mathrm{DP}=$ & Data di pubblicazione & Stringa ISO $|\mathrm{AAAA}|$ \\
\hline $\mathbf{T M}=$ & Tipo di materiale & $\begin{array}{l}\text { Stringa [esempio: TM=T } 200 \text { TESI } \\
\text { DI LAUREA] }\end{array}$ \\
\hline TT $=$ & Tipo di materiale & Parole \\
\hline $\mathbf{B} \mathbf{I}=$ & Biblioteca & Stringa [codice] \\
\hline$B C=$ & Biblioteca & Stringa [codice] \\
\hline $\mathrm{BB}=$ & Biblioteca & Parole \\
\hline $\mathbf{L} \mathbf{L}=$ & Lingua & Stringa \\
\hline $\mathbf{L N}=$ & Lingua & Parole \\
\hline $\mathrm{DB}=$ & Database & Stringa \\
\hline $\mathbf{S T}=$ & Tinlib & $\begin{array}{l}\text { ST=tinlib identifica i record importi } \\
\text { da tinlib }\end{array}$ \\
\hline $\mathrm{LO}=$ & Collocazione & $\begin{array}{l}\text { Stringa [Esempio: LO=BDPA } \\
\text { 1996/056 PAST] }\end{array}$ \\
\hline$I N=$ & Inventario & Stringa \\
\hline $\mathbf{C R}=$ & Bid & \\
\hline
\end{tabular}

Dove si intende per "Stringa" il contenuto intero del campo e per "Parole" le singole parole immesse in un campo.

I seguenti campi sono indicizzati in modo da essere anche visualizzati in OPAC come liste:

- Autore

- Relatore

- Titolo

- Collocazione

- Inventario 


\section{Digitalizzazione}

Il processo di digitalizzazione delle tesi ha avuto inizio nel 2000 esclusivamente per fini conservativi e per favorirne la fruizione. Sostanzialmente al precedente processo di microfilmatura delle tesi vecchie di più di dieci anni, si è sostituita la digitalizzazione. Il processo non ha alcun fine amministrativo, né di sostituzione degli originali, tuttora conservati in un deposito remoto, eventualmente accessibile su richiesta. Pertanto le tesi degli ultimi dieci anni sono sempre disponibili in formato cartaceo.

Non vengono digitalizzate le Tesi escluse dalla consultazione.

\section{Risorse umane impiegate}

Il responsabile dei servizi di digitalizzazione, impiegato al $50 \%$, per le attività di controllo, sovente onerose, nonché per le attività di salvataggio in formato prestabilito delle medesime.

Gli studenti 150 per l'attività di acquisizione tramite scanner².

\section{Stazione di digitalizzazione}

Sono disponibili le seguenti attrezzature per l'attività di acquisizione digitale:

- 2 personal computer con bus SCSI e $512 \mathrm{Mb}$. RAM collegati a ${ }^{3}$ :

- scanner planetario A2, toni di grigio e B\&W, 300 dpi

- scanner colore A3 1200 dpi

- fotocamera digitale

- scanner diapositive e positivi

${ }^{2}$ Oggi, a seguito dei tagli di bilancio, non sono più disponibili studenti 150 ore per queste attività. E' pertanto il personale interno che svolge l'attività di acquisizione.

${ }^{3}$ Oggi, viene utilizzato anche uno scanner con alimentatore automatico per le tesi spaginabili in formato A4 (solo B\&W). 


\section{Metodo di acquisizione}

Attività studenti 150 ore $^{4}$ :

- Acquisizione da scanner planetario per pagine toni di grigio o B\&W e scanner colore per pagine con colori ${ }^{5}$.

- Salvataggio in formato TIFF ${ }^{6}$.

- Integrazione dei file dei documenti a colori, di eventuali diapositive allegate acquisite con scanner per diapositive, di file di eventuali cdrom allegati (se possibile).

Attività personale IUAV:

- Acquisizione di formati particolari (fotocamera digitale)

- Controllo e salvataggio con "Acrobat" 5.0 in formato PDF.

\section{Formato oggetti digitali}

- File "TIFF" importati in "Acrobat".

- File "PDF" relativi a singola Tesi protetti da modifica e stampa.

- File "PDF" salvati su cd-rom con indicazioni elementari per la conservazione (nome file corrispondente a numero inventario)

\section{Documenti on-line}

I cd-rom vengono copiati su "thin server" "Axis". L'accesso da parte dei server "Linux" dell'OPAC EasyWeb al "thin server" avviene via "SAMBA"7. Il collegamento al database delle tesi avviene attraverso il numero inventario, uguale a nome del file "PDF".

${ }^{4}$ Oggi questa attività è svolta da personale interno.

${ }^{5}$ Oggi, nel caso di tesi spaginabili, utilizziamo lo scanner con alimentatore automatico.

${ }^{6}$ Oggi utilizziamo il formato TIFF multipagina per sequenze di pagine coerenti

${ }^{7}$ Oggi, a seguito di nuova release del sistema operativo dei "thin server" Axis, l'accesso avviene via "NFS", molto più veloce. 


\section{Accessibilità}

L'accesso è limitato agli IP relativi a:

- le stazioni multimediali con monitor 21 inch. disponibili agli utenti in sala consultazione riservata

- i PC degli operatori delle biblioteche dipartimentali.

Da altre postazioni IUAV è possibile solo tramite password specificatamente rilasciata.

\section{Limiti riscontrati}

Specifici, relativi alle tesi:

A tutt'oggi non è stato possibile ottenere direttamente gli originali in formato digitale. Sebbene la fornitura di tesi in formato digitale originale ridurrà sensibilmente le attività di scansione (restano quelle relative a formati particolari, a foto, etc.), si apriranno nuovi problemi, relativi ai formati in cui saranno fornite. Non è purtroppo ipotizzabile che tutte le tesi siano in formato "PDF". Gli studenti utilizzano sovente programmi che non producono direttamente i file "PDF". Sarà necessario prefigurare le attività di conversione in formati accessibili e gestibili nel tempo.

Generali, relativi agli “Oggetti digitali”:

- Le attrezzature utilizzate non sono ottimali, in parte obsolete.

- La Sicurezza non è certificata ed è demandata al livello locale.

- Manca una procedura OAIS che permetta la conservazione nel tempo nonché che garantisca l'accessibilità nel tempo delle tesi, mancano elementi di riconoscimento intrinseci alle tesi stesse (Metadati). Al momento è necessario il collegamento al database per risolvere il legame inventario/descrizione della tesi.

- La qualità dell'acquisizione è molto variabile in funzione del personale impiegato. 


\section{Sviluppi previsti}

È presumibile che prossimamente tutte le tesi vengano fornite in originale in formato digitale.

Sarà necessaria una progressiva sostituzione delle attrezzature obsolete.

Si auspica una centralizzazione della sicurezza, specificatamente per quanto riguarda le certificazioni e le login di accesso.

Dovremo utilizzare Metadati conservativi (procedure OAIS), gestionali, descrittivi. A tal fine è in corso una attività, supportata dalla consulenza della d.ssa Laura Anselmi, per definire le corrispondenze fra i campi utilizzati in EasyCat e i diversi Metadati disponibili. In particolare il formato "Dublin core" e il formato "MAG" predisposto dall'ICCU.

Auspichiamo l'introduzione di soluzioni di OPen ACcess che permettano l'accesso integrato a tutte le risorse locali e remote di interesse, tesi comprese.

Saranno infine prevedibili nuove soluzioni in relazione ai nuovi ordinamenti. 


\section{Tavola rotonda}

Si riassumono di seguito i punti salienti emersi nel corso della tavola rotonda:

1. Data la mancanza di leggi e regolamenti sull'argomento è di fatto perseguibile la via della cooperazione sulle regole, molto più che sulle indicazioni di software e hardware specifici, legati a troppe variabili locali della gestione, oltre che ad un mercato in continua evoluzione.

2. "Copyright" è concetto estraneo alla tradizione del diritto italiano: non contempla infatti i concetti per noi essenziali di "paternità intellettuale" e "diritto morale" dell'autore. Nel Copyright è implicito il concetto di "diritto di riproduzione", totalmente diverso. La pubblicazione scientifica oggi si trova in un circolo vizioso, che parte dal pagamento per la pubblicazione di un articolo su un periodico ad un editore, pagamento dell'abbonamento alla rivista da parte dell'Ateneo, ancora pagamento del copyright attraverso i più recenti provvedimenti di legge (accordi SIAE). Le vie per uscirne attualmente possono essere diverse: una di queste è la pubblicazione da parte degli autori su server appositi, come ad esempio a Los Alamos, ma questa soluzione non prevede interventi dei bibliotecari nella catena dell'informazione. Diversa la proposta del CERN, in cui invece i bibliotecari hanno parte diretta e importante nella gestione dell'archivio dei contributi dei ricercatori. Altra recentissima soluzione potrebbe essere quella degli "e-learning objects", passo di evoluzione degli Open Archives stessi. Infine, si potrebbe ipotizzare la nascita di una "University Press" per ogni Ateneo con compiti di diffusione anche solo digitale per questi materiali.

3. Anche le tesi di dottorato sono di difficile accesso e rientrano a pieno diritto nelle problematiche oggi esposte. Ricordiamo che il deposito obbligatorio presso la Biblioteca Nazionale Centrale di Firenze non è "pubblicazione" e che anche questi materiali presentano le stesse difficoltà delle tesi di laurea degli studenti. 
4. Recenti disposizioni legislative hanno depenalizzato alcuni aspetti del reato di copiatura della tesi, ma resta in vigore l'aspetto principale, relativo alla presentazione di elaborati falsificati. Le soluzioni proposte possono aiutare anche alla soluzione di questo problema, attraverso la diffusione di informazioni che rendano più difficile sottrarsi ad un confronto diretto dei testi presentati (Legge 19 aprile 1925 , n. 475 e recenti provvedimenti del Decreto Legislativo 30 dicembre 1999, n. 507). 


\section{Bibliografia}

Archivio generale di ateneo, Università degli studi di Padova (a cura di), Thesis 99: progetto per la gestione, tenuta e tutela delle tesi di laurea, <http://www.unipd.it/ammi/archivio/th_idx.htm>, ultimo agg. 2 giu. 1999. Padova, Università degli studi di Padova, 1996-1999

Associazione italiana biblioteche, Biblioteche speciali, "AIB notizie», 4 (1992) n. 11/12, p. 17

Associazione italiana biblioteche, Commissione Biblioteche aziendali, «AIB notizie», 4 (1992) n. 5, p. 10-11

Associazione italiana biblioteche, Commissione Biblioteche speciali e documentazione, «AIB notizie», 4 (1992) n. 2, p. 5-8

Associazione italiana biblioteche, Il Gruppo di studio "Tesi", "AIB notizie», 4 (1992) n. 6/7, p. 12

Borraccini Verducci, Rosa Marisa, I risultati di una indagine preliminare sul trattamento delle tesi nelle università italiane, in Alberani, Vilma - De Castro Pietrangeli, Paola (a cura di), La letteratura grigia. $1^{\circ}$ Convegno nazionale organizzato in collaborazione con l'Associazione italiana biblioteche, Istituto superiore di sanità, Roma, 4-5 giugno 1992, Roma, Istituto superiore di sanità, 1993, p. $173-178$

Borraccini Verducci, Rosa Marisa, Le tesi: un esempio di letteratura grigia, «Il bollettino CED», 2 (1992) n. 2, p. 2-3

Borraccini Verducci, Rosa Marisa - Verducci, Luigi, Una facoltà allo specchio: le tesi di laurea della Facoltà di lettere e filosofia dell'Università degli studi di Macerata (1964/65-1988/89), Firenze, Olschki, 1991

De Robbio, Antonella (a cura di), Diritto d'autore. La proprietà intellettuale tra biblioteche di carta e biblioteche digitali, Roma, Associazione italiana biblioteche, 2001 
Deandrea, Marina - Ferrero, Cristiana - Pellò, Barbara, Finalità e tecniche operative del progetto TEMT, «Bollettino d'informazioni / AIB», 27 (1987) n. 1, p. 35-43

Monaco, Giuseppina, Le tesi di diploma nella Scuola speciale per archivisti e bibliotecari e i rapporti con Current research, in Alberani, Vilma - De Castro Pietrangeli, Paola (a cura di), La letteratura grigia. $1^{\circ}$ Convegno nazionale cit., p. 169-172

Penzo Doria, Gianni, Primi appunti per la gestione, tenuta e tutela delle tesi di laurea, «Archivi \& computer», 8 (1998) n. 1, p. 9-24

Pistelli, Zanetta, Disponibilità e controllo bibliografico delle tesi: verso una normativa anche in Italia?, in Alberani, Vilma - De Castro Pietrangeli, Paola (a cura di), La letteratura grigia. $1^{\circ}$ Convegno nazionale cit., p. 165-168

Pistelli, Zanetta, La conservazione e la disponibilità delle tesi di laurea in un dipartimento universitario, «Il bibliotecario», n. 11/12 (mar.-giu. 1987), p. $171-178$

Pistelli, Zanetta, Problemi e prospettive per un archivio elettronico delle tesi italiane, In Alberani, Vilma - De Castro Pietrangeli, Paola (a cura di), La letteratura grigia. $3^{\circ}$ Convegno nazionale Istituto superiore di sanità, Roma, 25-26 novembre 1999, Roma, Istituto superiore di sanità, 2000, p. 132-140

Pistelli, Zanetta - Saliba, Michael Angelo, Breve descrizione del programma "Libra" per la gestione di tesi collocate in una biblioteca, «Il bibliotecario», n. 16 (giu. 1988), p. 129-134

Pistelli, Zanetta - Zanon, Antonio, La catalogazione delle tesi, Roma, Associazione italiana biblioteche, 2002

Revel, Lodovica, Sottocommissione Biblioteche aziendali, «AIB notizie», 5 (1993) n. 9, p. 17-18

Università degli studi di Padova (a cura di), Carta dei diritti delle tesi di laurea: bozza presentata alla $2^{a}$ Conferenza organizzativa degli archivi delle università italiane, <http://www.unipd.it/ammi/archivio/carta\%20dei\%20diritti \%20delle\%20tesi.htm>, ultimo agg. 15 nov. 1999, Padova, Università degli studi di Padova, 1996-1999. 
Venuda, Fabio, L'archiviazione elettronica delle tesi di laurea all'Università Ca' Foscari. Feb. 2001. «ESB forum», <http://www.burioni.it/forum/ firenze2001/fi01-venuda.htm>, anche in E.S. Burioni ricerche bibliografiche (a cura di), L'innovazione tecnologica ed organizzativa per $i$ servizi di biblioteca, Genova, E.S. Burioni ricerche bibliografiche, 2001, p.125-131

Zanon, Antonio, La catalogazione delle tesi: problematiche e ipotesi per una standardizzazione nelle università italiane, in Alberani, Vilma - De Castro Pietrangeli, Paola (a cura di), La letteratura grigia. $3^{\circ}$ Convegno nazionale cit., p. 78-83 


Stampato da:

Grafiche Cappelli srl Via Arno, 49 - Osmannoro

Sesto Fiorentino (FI) 